\title{
Formation of multiple populations in globular clusters: another possible scenario
}

\author{
A. A. R. Valcarce and M. Catelan \\ Pontificia Universidad Católica de Chile, Departamento de Astronomía y Astrofísica, Av. Vicuña Mackena 4860, $782-0436$ Macul, \\ Santiago, Chile \\ e-mail: [avalcarc, mcatelan] aastro.puc.cl
}

Received 24 March 2011 / Accepted 29 June 2011

\begin{abstract}
Aims. While spreads in chemical composition are now believed to be a universal characteristic of globular clusters (GCs), not all of them display evidence of multiple populations in their color-magnitude diagrams (CMDs). Here we present a new scenario for the formation of GCs, in an attempt to qualitatively explain these otherwise elusive observational results.

Methods. Our scenario divides GCs into three groups, depending on the initial mass $\left(M_{\mathrm{I}}\right)$ of the progenitor structure (PS), as follows: i) massive PSs can retain the gas ejected by massive stars, including the ejecta of core-collapse SNe. ii) Intermediate-mass PSs can retain at least a fraction of the fast winds of massive stars, but none of the core-collapse SNe ejecta. iii) Low-mass PSs can only retain the slow winds of intermediate-mass stars. The first group would contain members such as $\omega$ Centauri (NGC 5139), M 54 (NGC 6715), M 22 (NGC 6656), and Terzan 5, whereas NGC 2808 (and possibly NGC 2419) would be members of the second group. The remaining GCs that have only a spread in light elements, such as $\mathrm{O}$ and $\mathrm{Na}$, would be members of the third group.

Results. According to our scenario, the different components of $\omega$ Cen should not have a sizeable spread in age. We argue that this is consistent with the available observations. We present other simple arguments in favor of our scenario, which can be described in terms of two main analytical relations: i) one between the actual observed ratio of the number of first to second generation stars $\left(R_{\mathrm{SG}}^{\mathrm{FG}}\right)$ and the fraction of first generation stars that have been lost by the GC $\left(S_{\mathrm{L}}\right)$ and ii) another between $S_{\mathrm{L}}$ and $M_{\mathrm{I}}$. We also suggest a series of future improvements to and empirical tests of the modeling that may help decide whether the proposed scenario properly describes the chemical evolution of GCs.
\end{abstract}

Key words. globular clusters: general - stars: evolution - stars: abundances - stars: formation

\section{Introduction}

For many years, it was believed that globular clusters (GCs) follow with excellent precision the canonical zero-order approximation for the formation of simple stellar populations, where a chemically homogeneous gas cloud collapses under the action of its own potential well forming stars of different masses at the same time. However, recent observations have shown that this hypothesis is quite far from reality.

One of the peculiarities observed is a spread in the abundance of light elements in stars of all GCs studied to date. This phenomenon is very well represented by the O-Na anticorrelation (see Gratton et al. 2004, for a thorough review of the chemical composition of GC stars). This anticorrelation differs in detail from one GC to the next, and its extension seems to depend on the present-day GC mass but not on its metallicity (Carretta et al. 2010a).

Since massive GCs have deeper potential wells, it is commonly thought that a spread in Fe-peak elements would only be present in the most massive GCs. However, spectroscopic evidence has revealed heavy element variations in GCs less massive than others that do not display a spread in these elements. This is the case for both M 22 (NGC 6656) and NGC 1851, which have masses of $\sim 5.4 \times 10^{5} M_{\odot}$ and $\sim 5.6 \times 10^{5} M_{\odot}$ respectively, and heavy element variations, while other GCs (such as the extensively studied M 3, with a mass $\left.\sim 8.4 \times 10^{5} M_{\odot}\right)$ do not show this peculiarity ${ }^{1}$. While the stars in M 22 have a range of iron abundances between $[\mathrm{Fe} / \mathrm{H}] \approx-1.9$ and -1.6 , as well as spreads in other elements (Pilachowski et al. 1982; Lehnert et al. 1991; Da Costa et al. 2009; Marino et al. 2009, 2011b), NGC 1851 harbors two groups of stars with different $[\mathrm{Ba} / \mathrm{Fe}]$ ratios, but, depending on the authors, with a uniform $[\mathrm{Fe} / \mathrm{H}]$ (Yong \& Grundahl 2008; Villanova et al. 2010) or a spread in metallicity of $\Delta[\mathrm{Fe} / \mathrm{H}] \sim 0.2$ dex (Carretta et al. 2010b, 2011, who also associate NGC 1851 with a merger of two separate GCs). On the basis of a deep study of their color-magnitude diagrams (CMDs), it has been shown that for these GCs the simple stellar population hypothesis is ruled out, as revealed by an observed subgiant branch (SGB) split (Milone et al. 2008; Marino et al. 2009).

Another GC for which an SGB split has been found is NGC 6388 (Piotto 2009), which has a mass of $1.4 \times 10^{6} M_{\odot}$. Cassisi et al. (2008) pointed out that such SGB splits might be related to an enhancement in $\mathrm{CNO}$ abundances, or an internal age difference of a few Gyr. However, Villanova et al. (2010) have shown that for NGC 1851 the $\mathrm{C}+\mathrm{N}+\mathrm{O}$ content would be constant between the two groups with different $[\mathrm{Ba} / \mathrm{Fe}]$. Moreover, Di Criscienzo et al. (2010) pointed out that these SGB splits may

\footnotetext{
${ }^{1}$ Here, as in the rest of this paper, we estimate the mass of each GC using $M_{V}$ from the Harris (1996, revision 2010) catalog and a mass-tolight ratio $M / L_{V}=3 M_{\odot} L_{\odot}^{-1}$, as predicted by simple stellar population models (Bruzual \& Charlot 2003).
} 
also be caused by variations in the helium abundance, though only at moderately high metallicity.

For the moment, NGC $2808\left(1.4 \times 10^{6} M_{\odot}\right)$ seems to be a fairly special case, with at most a small spread in metals $([\mathrm{Fe} / \mathrm{H}]=-1.10 \pm 0.03)$ but a wide $\mathrm{O}-\mathrm{Na}$ anticorrelation (Carretta et al. 2006). This GC has three populations observed down to the main sequence (MS), which suggests that it contains a range of helium abundances, from $Y=0.25$ to $Y=0.37$ (Norris 2004; D'Antona et al. 2005; Piotto et al. 2005, 2007). This last property was predicted by D'Antona et al. (2002) from a study of the cluster's horizontal branch (HB) morphology, and tested by Dalessandro et al. (2011) using high-resolution far-UV and optical images, as well as Pasquini et al. (2011) using visual and near-IR spectroscopy. Another GC that may share properties similar to those of NGC 2808 is NGC 2419, with a presentday mass of $1.4 \times 10^{6} M_{\odot}($ Di Criscienzo et al. 2011; Caloi \& D'Antona 2011; see also Cohen et al. 2010).

On the basis of photometry, the case of the most massive Galactic GC $\left(3 \times 10^{6} M_{\odot}\right), \omega$ Cen, has been known for a long time (Geyer 1967; Cannon \& Stobie 1973), although only recent studies have unveiled some of its most intriguing properties. This GC has at least three separate, well-defined red giant branches (RGBs), an extended HB morphology, and a large number of subluminous extreme HB stars (e.g., D'Cruz et al. 1996, 2000; Whitney et al. 1998; Pulone et al. 1998; Pancino et al. 2003; Rey et al. 2004; Freyhammer et al. 2005; Cassisi et al. 2009; Bellini et al. 2009; Calamida et al. 2009, 2010). Hubble Space Telescope (HST) observations have also uncovered a triple MS (Bedin et al. 2004; Bellini et al. 2009, 2010), in addition to at least four SGBs (Villanova et al. 2007). On the basis of spectroscopic studies, this GC is also known to have a complex behavior. From spectroscopically derived chemical compositions of MS stars, Piotto et al. (2005) suggested that the bluest MS is highly enriched in helium $(Y=0.35)$, while metallicities of SGB and RGB stars have a wide distribution, from $[\mathrm{Fe} / \mathrm{H}] \sim-2$ to $\sim-0.4$ (Hilker et al. 2004; Villanova et al. 2007; Johnson et al. 2009; Johnson \& Pilachowski 2010, and references therein). These different metallicity groups have some intriguing properties, including a smaller spread in the abundances of some elements (e.g., C, N, Ca, Ti, and Ba; see Fig. 16 in Villanova et al. 2007) at the more metal-rich end, compared to metal-poor stars in the cluster. It would thus appear that the metal-rich stars were all formed at the same time, from a fairly chemically homogeneous cloud. Metal-poor stars may also all have formed at the same time, but from a chemically inhomogeneous cloud. Alternatively, these metal-poor stars may have formed at different times from material processed in different ways (see also Gratton et al. 2011).

Another GC with properties similar to $\omega$ Cen's, albeit at a much less extreme level, is M $54\left(2.3 \times 10^{6} M_{\odot}\right)$, in the nucleus of the Sagittarius dwarf galaxy (e.g., Siegel et al. 2007). That M 54 is the second most massive GC in the Galactic neighborhood strongly suggests that $\omega$ Cen may also be the remnant of a formerly much larger structure (e.g., Dinescu 2002; Altmann et al. 2005; Wylie-de Boer et al. 2010, and references therein). Other GCs for which internal dispersions in the Fe-peak elements have been detected, and that may thus share some similarities in their chemical evolution with $\omega$ Cen and M 54, include M 22 (e.g., Da Costa et al. 2009; Marino et al. 2009, 2011b) and Terzan 5 (Ferraro et al. 2009; D'Antona et al. 2010; Origlia et al. 2011).

An intriguing GC is 47 Tucanae (NGC 104), which in spite of being one of the most massive Galactic GCs $\left(1.4 \times 10^{6} M_{\odot}\right)$ has only a moderately wide SGB - a feature that is interpreted by Di Criscienzo et al. (2010) as evidence of subpopulations of slightly different helium abundances, instead of an internal spread in either age or $\mathrm{C}+\mathrm{N}+\mathrm{O}$. Importantly, the lack of prominent subpopulations in 47 Tuc suggests that the present-day GC mass does not correlate tightly with either the presence or extent of the multiple populations observed in GC CMDs, which probably indicates that there has been significant mass evolution in these objects (e.g., Vesperini 1998; Fall \& Zhang 2001; Bekki \& Norris 2006; D’Antona et al. 2007; McLaughlin \& Fall 2008; Conroy 2011, and references therein). On the other hand, Nataf et al. (2011) found a radial gradient in the luminosity of the RGB bump in 47 Tuc, which they interpreted as evidence of a second generation of stars produced deep within the cluster's potential well.

In this paper, we present a scenario that tries to explain qualitatively the observed CMD peculiarities, where the main parameter that defines the present-day CMD is the initial mass $\left(M_{\mathrm{I}}\right)$ of the progenitor structure (PS), as opposed to the GC's presentday mass $\left(M_{\mathrm{GC}}\right)$. We assume that all GCs have lost an unknown amount of mass that is not necessarily related with $M_{\mathrm{GC}}$, but can be estimated assuming a simple analytical model.

Our paper is structured as follows. First, we review in Sect. 2 some scenarios for the formation of GCs. This is followed in Sect. 3 by an explanation of our proposed scenario. Then in Sect. 4 we estimate the $M_{\mathrm{I}}$ value of the PS that in our scenario has given birth to the present-day GC, based on the observed ratio of the number of second to first generation stars $\left(R_{\mathrm{FG}}^{\mathrm{SG}}\right)$. In this section, we also discuss some open problems with our scenario. Finally, in Sect. 5 we summarize our main results.

\section{Previous scenarios}

For decades, there have been many attempts to establish a scenario to explain the formation and evolution of GCs (e.g., Matsunami et al. 1959; Pikel'Ner 1976; Di Fazio \& Renzini 1980; Di Fazio 1986; Tenorio-Tagle et al. 1986; Brown et al. 1991; Murray \& Lin 1992; Price \& Podsiadlowski 1993; Richtler \& Fichtner 1993; Murray \& Lin 1993; Brown et al. 1995; Salaris \& Weiss 1997; Nakasato et al. 2000), but only recently has it become clear that the common assumption that they are simple stellar populations (SSP) is generally incorrect - and, importantly, not only in the well-known cases of $\omega$ Cen and (more recently) M 54. There is increasing evidence that virtually all GCs show some level of chemical composition inhomogeneity (e.g., Carretta et al. 2009a,b), which in some cases may lead to multiple sequences in the observed CMDs. In what follows, we summarize some of the scenarios that have been proposed to explain the new empirical evidence (see Renzini 2008, for a more detailed comparison).

\subsection{Decressin et al. (2007a) scenario}

Decressin et al. (2007a) presented a scenario to explain the multiple populations observed in some massive GCs that invokes pollution from rapidly rotating massive stars (FRMS, Decressin et al. 2007b). These are stars that have reached the critical rotation velocity, and formed an equatorial gas disk around them. Unlike the normal winds of massive stars, which have velocities ranging from hundreds to a few thousand $\mathrm{km} \mathrm{s}^{-1}$, the equatorial disk loses mass with velocities lower than $50 \mathrm{~km} \mathrm{~s}^{-1}$, that can then easily be retained by the potential wells of GCs. In this scenario, FRMS ejecta are mixed with pristine gas to form second generation (SG) stars near their massive progenitors. Even though this scenario may help us to explain the O-Na anticorrelation observed in all GCs, the multiple MSs observed in some 
GCs cannot be explained without invoking discrete helium abundances, which is in conflict with the smooth spread in $Y$ predicted by this scenario, as also pointed out by Renzini (2008).

\subsection{D'Ercole et al. (2008) scenario}

In D'Ercole et al. (2008), the formation of multiple populations is attributed to the gas ejected by intermediate-mass AGB (or "super-AGB") stars (D'Antona et al. 1983; Ventura et al. 2001, 2002; Thoul et al. 2002), with this gas starting to collect in the GC core after the last core-collapse SN explosion. In this sense, the formation of a cooling flow is studied by the authors using one-dimensional hydrodynamical simulations. Such a cooling flow collects the gas expelled by super-AGB stars that is used to form the SG stars. Using $N$-body simulations, the authors study the dynamical evolution of both populations, showing that if SG stars are formed in the center of the GC, most of the expelled stars will be first generation (FG) stars, which can result in a GC dominated by SG stars.

For their "standard" scenario, hydrodynamical simulations show that the star formation rate increases since $10 \mathrm{Myr}$ (the time of the last core-collapse SN) until $40 \mathrm{Myr}$ (the time of the first SN Ia). However, the effect of core-collapse SNe belonging to the SG, which must stop the formation of SG stars after a few Myr, is not considered.

We also note that every FG star that does not explode as a core-collapse $\mathrm{SN}$ is a possible progenitor of a type Ia SN. The more massive the progenitor, the closest the remaining white dwarf will be to the Chandrasekhar mass (e.g., Salaris et al. 2009; Kalirai et al. 2009). Therefore, after the core-collapse $\mathrm{SNe}$ period, there must also be a period of type Ia SNe, which will inhibit the formation of stars with gas ejected by more massive intermediate-mass stars. Naturally, the frequency of these events must decrease with time, instead of remaining constant. In our opinion, this poses considerable problems for the explanation of multiple populations with high helium abundances in GCs using intermediate-mass stars.

\subsection{Conroy \& (I2011) scenario}

In the Conroy \& Spergel (2011) scenario, the FG stars are born in an intracluster medium previously enriched in metals (presentday GC metallicity), while after some Myr the remaining gas is completely expelled by core-collapse SNe. The GC is then ready to start the accretion of the intermediate-mass AGB ejecta for several $\times 10^{8} \mathrm{yr}$. During this time, the GC is also accreting pristine material from the ambient interstellar medium (ISM), which mixes with the AGB ejecta to form SG stars. Finally, SG corecollapse SNe, and later FG type Ia SNe, begin to explode, keeping the GC gas-free, hence curtailing the star formation process.

Even though the accretion of material from the ISM is plausible, it is unlikely that the chemical composition of this accreted material is similar to that of the GC itself. Moreover, if corecollapse SN explosions clean the intracluster medium, they must also clean the surrounding ISM or/and increase its metallicity, implying that SG stars will be formed with different metal abundances.

To close, we note that D'Ercole et al. (2011) also argued against this scenario, pointing out in particular that it is not possible to form very O-poor stars through the process envisaged by Conroy \& Spergel (2011). D'Ercole et al. also pointed out, following Renzini (2008), that the Conroy \& Spergel scenario also fails to account for the large amount of He that is needed to explain the highly He-enriched populations that appear to be present in some GCs (see Sect. 1).

\subsection{Carretta et al. (2010a) scenario}

Carretta et al. (2009b, and references therein) made a remarkable effort to obtain a homogeneous spectroscopic database for 17 GCs, which they studied focusing mainly on the O-Na anticorrelation. These data were used to divide the stellar populations in GCs into three components: i) a primordial population, containing stars with $\mathrm{O}$ and $\mathrm{Na}$ abundances similar to field stars (i.e., stars with $[\mathrm{Na} / \mathrm{Fe}]<[\mathrm{Na} / \mathrm{Fe}]_{\min }+0.3$, where $[\mathrm{Na} / \mathrm{Fe}]_{\min }$ is the lowest $\mathrm{Na}$ abundance detected in that $\mathrm{GC}$ ); ii) an intermediate population, containing stars with $[\mathrm{O} / \mathrm{Na}]>-0.9$ that do not belong to the primordial population, amounting to $60 \%$ to $70 \%$ of the observed stars; and iii) an extreme population, containing stars with $[\mathrm{O} / \mathrm{Na}]<-0.9$, which are not present in all GCs.

Using these data and other global parameters characterizing the GCs in their sample, Carretta et al. (2010a) proposed a scenario where GCs were formed in three different stages. First, a precursor population of stars is formed when the unborn GC (with a size of $\sim 100 \mathrm{pc}$, consisting of gas and dark matter) interacts strongly with other structures. Core-collapse $\mathrm{SNe}$ of this population enrich the remaining gas, and trigger the formation of the primordial population. The gas ejected by primordial FRMS or super-AGB stars give rise to a gas cloud chemically enriched in the center, where the second generation (SG) of stars is born. Finally, SG core-collapse SNe clean the remaining gas, thus halting star formation. During this time, the structure has lost all its dark matter content, almost all the precursor stars, and a large fraction of the primordial population.

While this appears to provide a promising framework, there are some points that require a closer inspection. Primordial lowmass stars, which are not observed in the actual GC because they were expelled from the initial structure, must still be present somewhere. Therefore, according to this scenario, a large number of metal-poor stars must be present in the field. However, to date there are only 174 known stars with $[\mathrm{Fe} / \mathrm{H}] \leq-3$, and 659 stars with $[\mathrm{Fe} / \mathrm{H}] \leq-2$ (SAGA database, ${ }^{2}$ Suda et al. 2008). Alternatively, the initial mass function (IMF) must be top-heavy at low metallicities (Skillman 2008; Komiya et al. 2010). We note, in addition, that if the gas ejected by the precursor corecollapse $\mathrm{SNe}$ is retained in the initial structure, the ejecta of the primordial core-collapse $\mathrm{SNe}$ must also be retained, which would increase the metallicity of the SG stars unless the initial structure had already lost a large fraction of its mass in a short period of time.

\subsection{An argument against the super-AGB scenario for the population richest in $\mathrm{He}$}

D'Ercole et al. (2010) demonstrated that, to reproduce the observed O-Na anticorrelation, as well as the suggested He spread in NGC 2808, SG stars must form only from super-AGB ejecta, whereas in the case of M 4 (NGC 6121) SG stars must be formed with a large amount of pristine gas mixed in. This is very surprising because NGC 2808 is ten times more massive than M 4 $\left(1.8 \times 10^{5} M_{\odot}\right)$, whereas it should be easier for a massive PS than for a low-mass PS to retain pristine gas. In this case, M 4 would have lost many more stars since its formation than NGC 2808 has, implying a more massive PS in the past - but not so massive as to have retained any (unobserved) trace of metals from

2 http://saga.sci.hokudai.ac.jp 


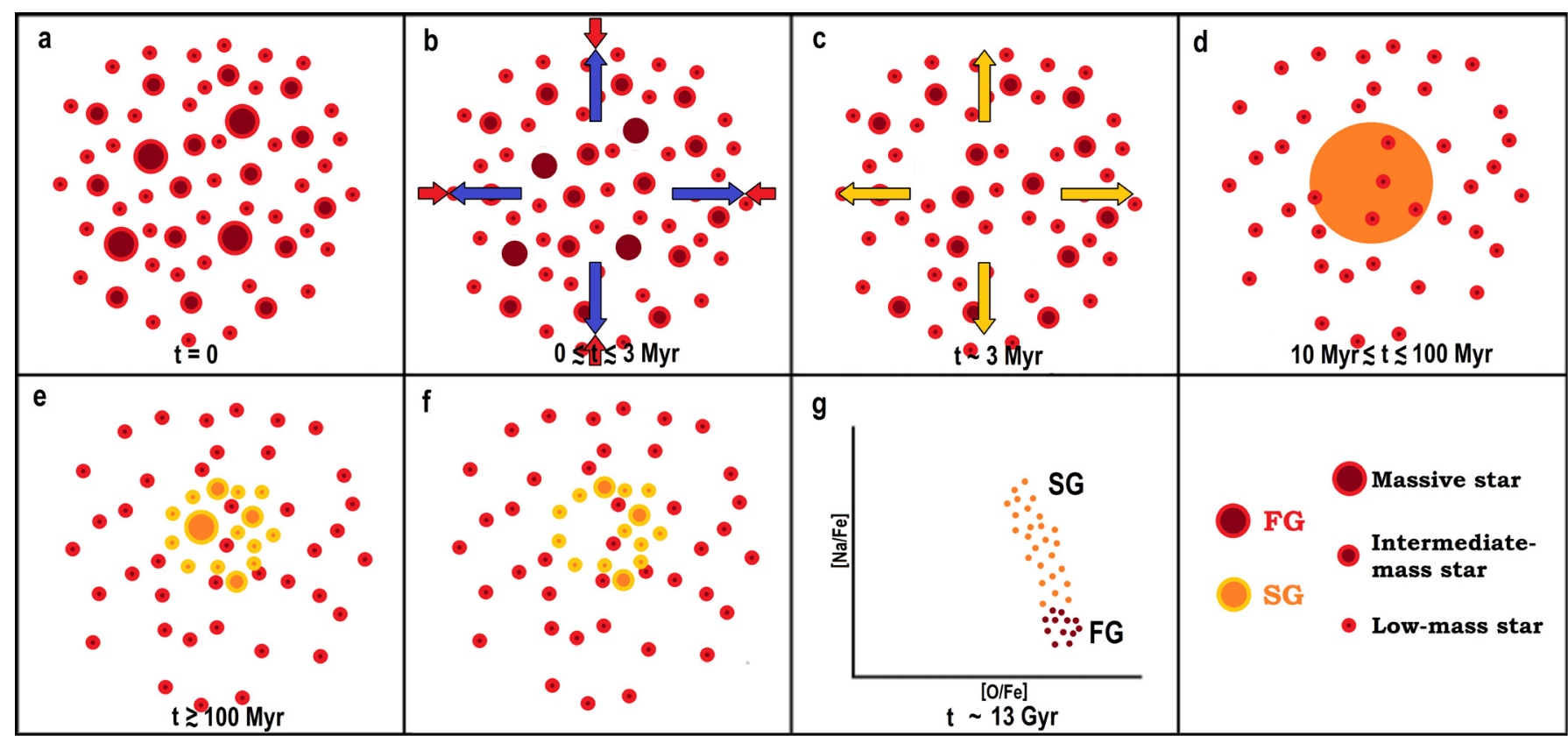

Fig. 1. Schematic representation of our scenario for low-mass PSs. Stars of the first and second generations are represented as red and orange, respectively. Sizes indicate the different star masses, where large, medium, and small circles represent massive, intermediate-mass, and low-mass stars, respectively. Arrows show the gas flux, with the arrow color indicating the chemical composition following the same color scheme as for the stars of the different generations, and the arrow size being roughly proportional to the gas speed. Colored areas represent zones of gas accumulation. In each panel, an approximate time for these events is given. Panels represent: a) formation of FG stars; b) pristine gas expulsion due to winds of FG massive stars; c) core-collapse SN ejecta are not retained in low-mass PSs; d) gas accumulation from winds of FG super-AGB and AGB stars; e) formation of SG stars; f) SG core-collapse SNe period; g) present-day O-Na anticorrelation. This figure is available in color in electronic form.

SN explosions. However, owing to their deeper potential wells, massive PSs are expected to lose a smaller number of stars than less massive PSs. This is contrary to what would have been expected if M 4 were related with a massive PS but NGC 2808 with a less massive one, unless the M 4 progenitor has been affected by stronger dynamical interactions in the course of its lifetime.

Finally, we note that, in our scenario, super-AGB and/or normal AGB stars do play an important role in the formation of multiple populations. As we will show, while our scenario implies that these stars cannot be the progenitors of the most heliumrich population observed in NGC 2808 and $\omega$ Cen, it does also indicate that they are likely to be the progenitors of the stars with helium abundances falling in-between those of the "normal" (i.e., non-He-enriched) and most He-rich stars.

\section{Our scenario}

Our new scenario is divided in two stages, and is schematically described in Figs. 1, 3, and 5. The first stage lays the foundations for the formation of any GC, reflecting the conditions in the ISM that allow a gravitationally bound "proto-GC" to form (panels labeled "a" in Figs. 1, 3, and 5). In turn, the second stage depends on the PS initial mass $M_{\mathrm{I}}$, and can have three different outcomes. More specifically, i) a low-mass PS only retains the mass ejected via slow winds from intermediate-mass stars; ii) an intermediate-mass PS does not retain the core-collapse $\mathrm{SNe}$ ejecta, but does retain at least a fraction of the wind from massive stars; and iii) a massive PS retains the winds of massive stars, and also the ejecta of core-collapse SNe.

\subsection{First stage}

Our envisaged first stage takes place as follows.

1. As usual, the first step in the formation of a GC begins with the gravitational collapse of a cloud with the present-day metallicity of the GC.

2. FG stars are then formed following a homogeneous distribution throughout the GC. They are initially embedded inside the ISM gas that was not used up to form stars (panels labeled "a" in Figs. 1, 3, and 5).

3. Assuming a star formation efficiency $(\epsilon)$ of between $20 \%$ and $40 \%$ (Parmentier et al. 2008), the remaining gas has a mass of between $0.8 M_{\mathrm{I}}$ and $0.6 M_{\mathrm{I}}$, but is distributed over a larger volume than required to trigger a new local fragmentation process to form a new generation of stars. This implies a decrease in the local gas pressure for the same potential well, with the consequence that the remaining gas starts to fall again into the center of the cluster.

4. The final step of this stage begins when massive stars eject at high velocities their envelopes, which then collide with the falling gas. This leads to a decrease in the speed of the expanding massive star ejecta, and to a decrease in the speed of the infalling gas (panels labeled "b" in Figs. 1, 3, and 5). The chemical composition of the massive star ejecta is initially quite similar to the primordial one, although after a while (depending on the initial stellar mass) this ejecta consists almost entirely of helium (Limongi \& Chieffi 2007), without any variations in heavy element abundances except mainly for those elements that participate in the $\mathrm{CNO}, \mathrm{NeNa}$, and $\mathrm{MgAl}$ cycles. 


\subsection{Second stage for low-mass PSs}

For low-mass PSs, the second stage takes place as follows (see also panels a through $\mathrm{f}$ in Fig. 1):

5a. Low-mass PSs are unable to retain the FG massive star ejecta because of their shallower potential well, and the consequent low velocity of the infalling primordial gas. For the same reason, the PS core does not contain enough material to transform all the kinetic energy of massive star ejecta into thermal energy. The formation of a viable star-forming cloud in the core is thus inhibited (panel b in Fig. 1).

6a. The next step is driven by the FG core-collapse SNe explosions, which completely clean the PS of the remaining primordial gas (panel c in Fig. 1).

7a. In this case, SG stars are formed only with (diluted) gas ejected by super-AGB and/or AGB stars (panels d and e), with the chemical composition of the ejecta depending in detail on the stellar mass.

8a. This is potentially a continuous process, with renewed cleansing of the intracluster gas taking place after each new star formation event (panel $\mathrm{f}$ in Fig. 1).

If the above scenario is correct, each successive stellar generation would leave a different signature in frequently observed diagnostic planes, such as the $[\mathrm{Na} / \mathrm{Fe}]$ versus (vs.) $[\mathrm{O} / \mathrm{Fe}]$ diagram. This diagram is schematically shown in panel $\mathrm{g}$ of Fig. 1. The anticipated impact on the HR diagram is shown in Fig. 2, where our new set of Princeton-Goddard-PUC (PGPUC) isochrones has been used (Valcarce et al., in prep.).

\subsection{Second stage for intermediate-mass PSs (NGC 2808's progenitor)}

A schematic representation of the second stage of the formation of intermediate-mass PSs, which are related to the progenitors of GCs such as NGC 2808 (and possibly NGC 2419), is shown in panels $\mathrm{b}$ through $\mathrm{f}$ of Fig. 3, and can be summarized as follows:

5b. Since intermediate-mass PSs have deeper gravitational potential wells than low-mass PSs, the infalling gas reaches a higher speed, and a fraction of the massive star ejecta is retained before their progenitors explode. However, in the outer parts of the PS the massive star ejecta are trying to escape, as the pristine gas is infalling (panel b in Fig. 3). Additionally, in the PS core, where the primordial gas has been accumulating, the gas ejected by massive stars is slowed down, and the kinetic energy transformed into thermal energy, which delays further star formation.

6b. When FG core-collapse SNe explode, their ejecta compress the center cloud, thus triggering star formation in the SG. These SG stars have been highly enriched in helium by the massive star ejecta, but at the same time are not heavily enriched in metals. Assuming that SN explosions are nearly symmetrical and that their precursors are not too close to the center, only a small fraction of the SN ejecta, which is metal-enriched, will be mixed with the core cloud. This event also completely removes the outer mixed gas from the cluster (panel c in Fig. 3), since the gravitational potential well is not deep enough and the mass of the outer infalling gas is insufficient to retain the $\mathrm{SNe}$ ejecta. Thus, TG stars will accordingly not be metal-enriched.

7b. The ejecta of massive SG stars are not retained in the case of an intermediate-mass PS, because the infalling gas is only produced by intermediate-mass FG stars. If any such gas is

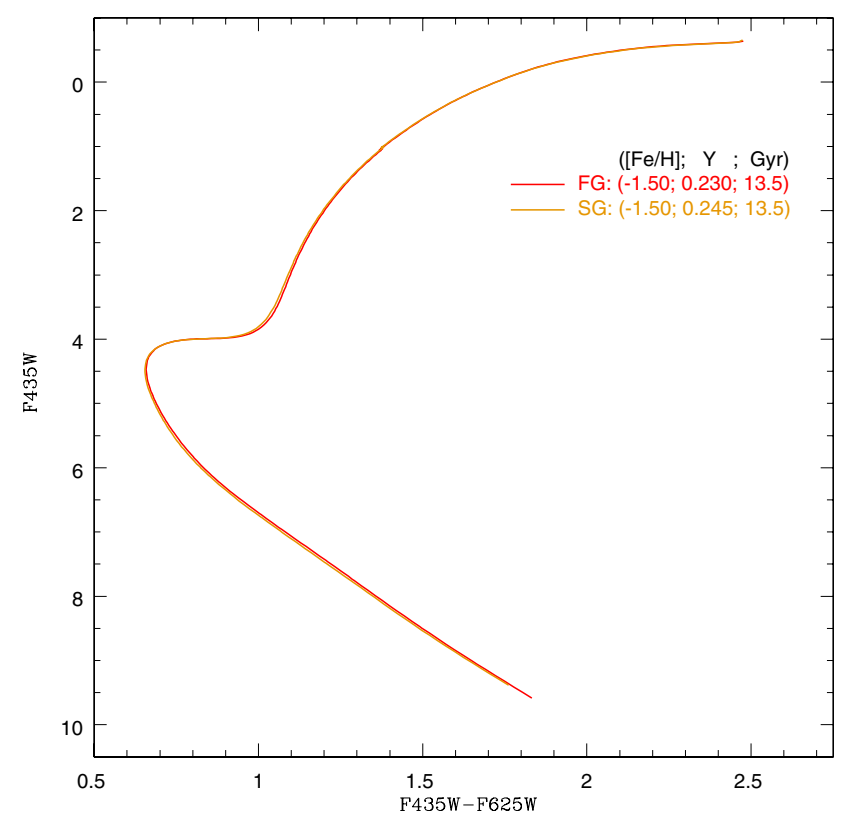

Fig. 2. CMD of a low-mass PS after 13 Gyr. PGPUC isochrones represent the two surviving populations for this system: FG (red lines) and SG (orange lines). This figure is available in color in electronic form.

initially retained, it will eventually be expelled by SG corecollapse SNe or FG type Ia SNe explosions, or will form but very few stars (panel d in Fig. 3).

8b. After this second cleansing of the cluster, a new cloud begins to form in the cluster center, using the intermediatemass stellar ejecta (mass lost at low velocity) from FG and SG stars (panel e in Fig. 3). The chemical composition of this new cloud falls between both generations, as a consequence of the slope of the IMF (which favors low-mass stars) and the mass ratio of both generations. Here, the first stars of the third generation (TG) will be created.

9b. These star formation and cluster ISM cleansing stages continue, with each successive stellar population becoming chemically more similar to FG stars, while at the same time less numerous (panel $\mathrm{f}$ in Fig. 3).

In panel g of Fig. 3, we show schematically the expected shape of the diagnostic $[\mathrm{Na} / \mathrm{Fe}]$ vs. $[\mathrm{O} / \mathrm{Fe}]$ diagram. The corresponding HR diagrams for this class of PS are shown in Fig. 4.

\subsection{Second stage for massive PSs ( $\omega$ Cen's progenitor)}

Figure 5 is a schematic representation of our scenario for massive PSs, which is explained in the following paragraphs. Here, one can identify $\omega$ Cen as a prototype of massive PSs - but other possible examples include M 22, M 54, and Terzan 5 (see Sect. 1).

$5 c$. As in $5 b$, in the PS core a cloud has been formed that is highly enriched in helium. Its material comes from massive star ejecta and a fraction of the remaining gas that was not used to form FG stars. However, in the outer part of the PS the massive star ejecta is trying to escape, while the pristine gas is infalling to the PS center with a velocity (and a total mass) that is higher than in the case of intermediatemass PSs.

6c. The first core-collapse $\mathrm{SNe}$ explosions compress the core gas, triggering the SG star formation episode (panel c in Fig. 5). As in 6b, these SG stars are highly enriched in helium 


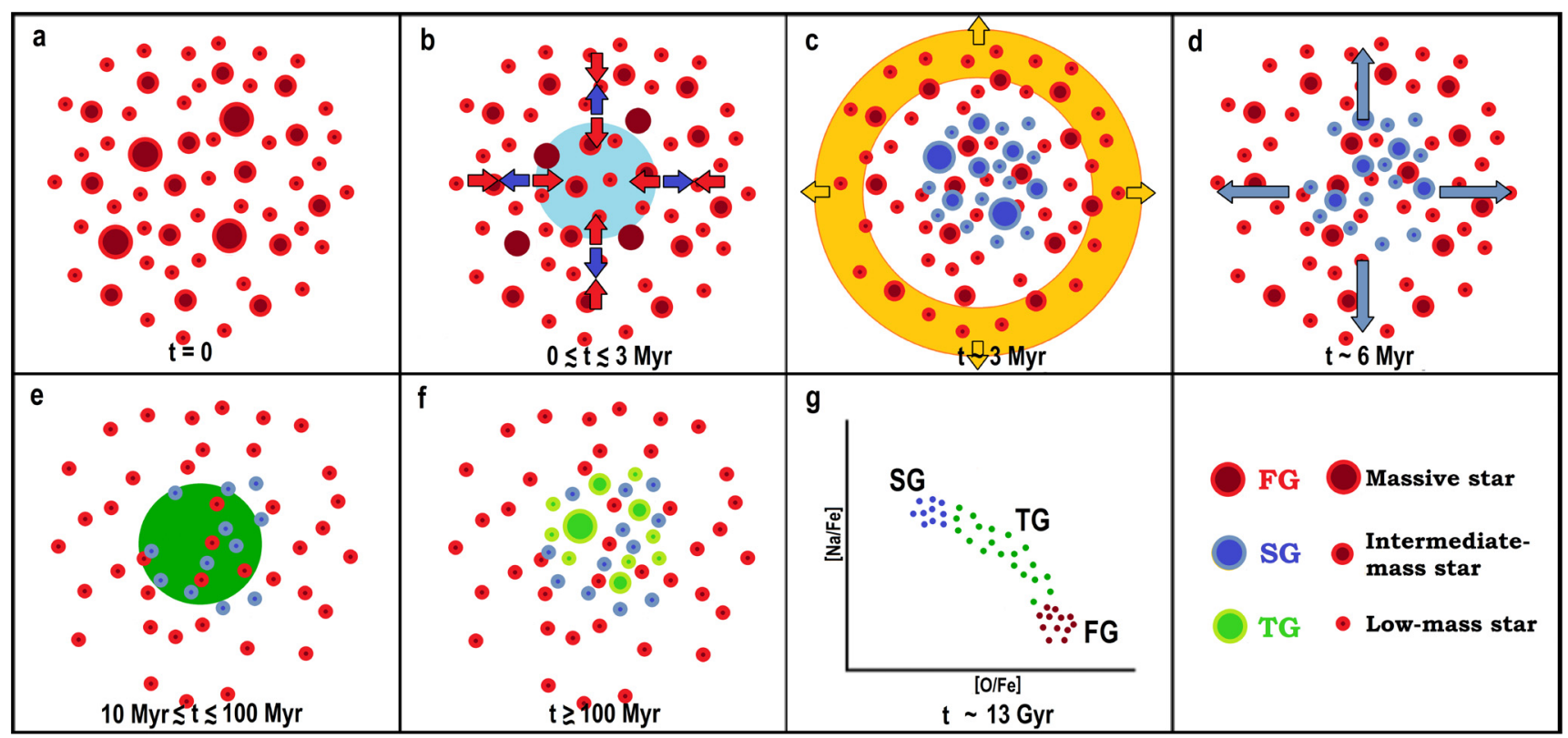

Fig. 3. As in Fig. 1, but for intermediate-mass PSs. Here the first, second, and third generation of stars are represented in red, blue, and green, respectively. Panels represent: a) formation of FG stars; b) gas accumulation from winds of FG massive stars and pristine gas; $\mathbf{c}$ ) first core-collapse SN explosions, which trigger the formation of SG stars and expel the gas that has not yet arrived at the core; d) FG and SG SN explosions; e) gas accumulation from winds of super-AGB and AGB stars of the FG and SG; f) formation of TG stars; g) present-day O-Na anticorrelation. This figure is available in color in electronic form.

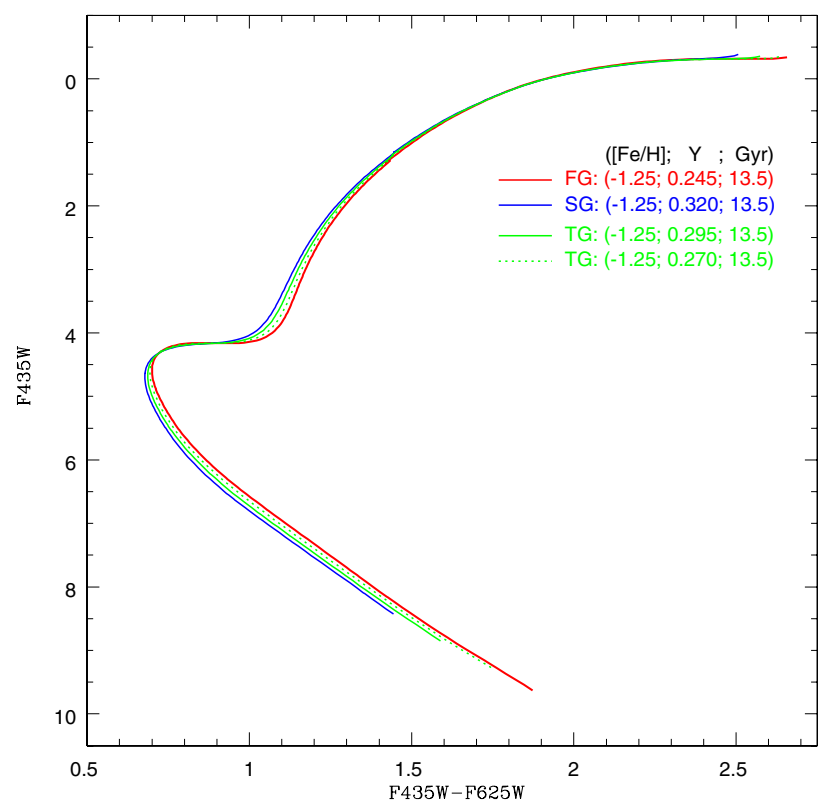

Fig. 4. As in Fig. 2, but for an intermediate-mass PS. PGPUC isochrones represent the different populations presented for this PS: FG (red lines), SG (blue lines), and TG (green lines). This figure is available in color in electronic form.

(without however having significantly higher metal abundances). However, in contrast to what happens in the case of intermediate-mass PSs, the deeper potential well does allow the gas in the outer part of the cloud to be retained. Most of the SN ejecta tries to escape the cluster, merging with the infalling gas in the process. This event efficiently mixes both gas components, and delays the moment of arrival of this mixed gas to the core.

7c. After a while, the highly metal-enriched material is mixed with the gas that was not used to form SG stars, creating a new cloud in the core of the cluster (panel d in Fig. 5). This cloud is also fed by SG massive stars and by both massive and intermediate-mass FG stars - and these provide the chemical ingredients that will characterize the cluster's third generation (TG) of stars (panel e in Fig. 5). Unfortunately, this population has a helium abundance that is difficult to predict, because of the several sources of material that are involved. However, we expect that TG stars have some degree of helium enrichment.

$8 \mathrm{c}$. Now, with three stellar generations in the cluster, the process of star formation continues, but each time with material processed mainly by less massive stars (super-AGB and/or AGB stars). As a result, the newborn stars belonging to the fourth generation $(4 \mathrm{G})$ will have a chemical composition that is a mixture of products from the three preceding generations. However, in our scenario each new star that is formed will have a chemical composition more similar to that of the primordial gas, owing to two main factors: i) the IMF slope, which implies that more stars with low masses are formed than massive stars; and ii) the relatively small amount of mass used to form each star generation. In our scenario, SG and TG stars are created using only a relatively small fraction of the total mass that was used to form FG stars. In other words, subsequent stellar generations will be increasingly affected by the evolution of lower-mass stars, whose ejecta will not be chemically very different from that of the original PS gas (panels $f$ and $g$ in Fig. 5).

9c. Finally, a massive PS can lose the gas that has not been used to form stars in different ways, the most important mechanism probably being the interaction with larger structures - e.g., when a PS passes through the Milky Way. When this happens, at first only the intracluster gas is lost to the Galaxy $^{3}$. Subsequently, however, owing to the decrease in

3 According to Priestley et al. (2011), the mass evolution of the intracluster gas depends mainly on the PS mass, the PS velocity, the specific stellar mass loss rate, and the density of the medium where the PS is moving. However, to properly test our scenario, an extension of their 


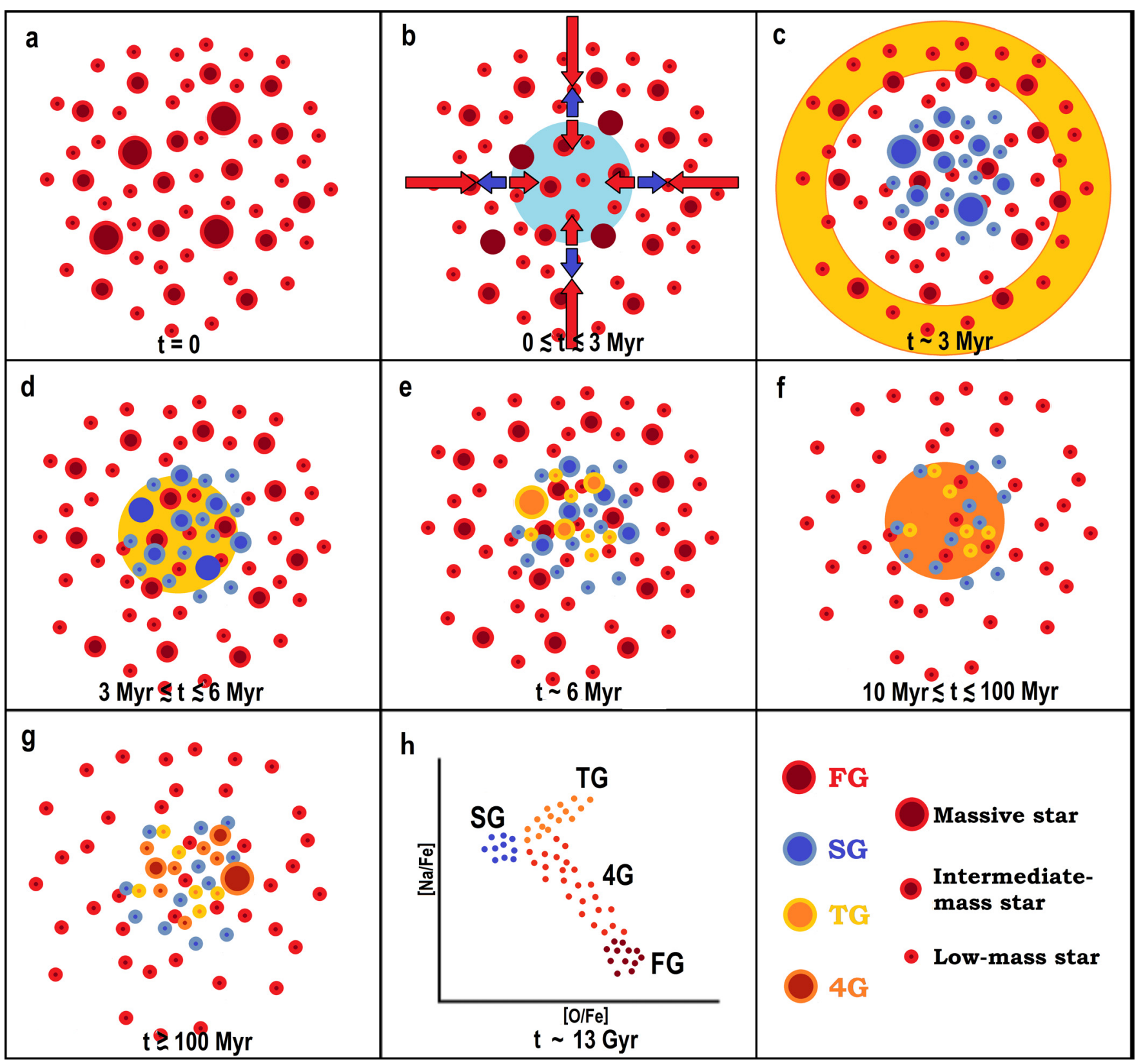

Fig. 5. As in Fig. 1, but for massive PSs. Stars of the first, second, third, and fourth generations are represented as red, blue, yellow, and orange circles, respectively. Panels represent: a) formation of FG stars; b) gas accumulation, from a mix of winds of FG massive stars plus pristine gas; c) first core-collapse SN explosions, which trigger the formation of SG stars; d) gas accumulation, from a mix of FG SN ejecta, winds of FG and SG massive stars, and gas remaining from the formation of SG stars; e) formation of TG stars, when SN explosions end; f) gas accumulation, from winds of super-AGB and AGB stars from all generations; $\mathbf{g}$ ) formation of $4 \mathrm{G}$ stars; $\mathbf{h}$ ) present-day $\mathrm{O}-\mathrm{Na}$ anticorrelation. This figure is available in color in electronic form.

the PS's potential well, the sizes of the stellar orbits increase (e.g., Moeckel \& Bate 2010; Decressin et al. 2010; Trenti et al. 2010, and references therein), and in this way the massive PSs can also more easily lose their stars. If the gas expulsion is inefficient - for instance, when the massive PS has a lower interaction with larger structures - the PS does not lose a large number of stars because the gas has been retained, thus ending up as a dwarf galaxy.

The strength of the interaction of course depends on the mass of the host galaxy. In the case of smaller galaxies (e.g., the Large

study to higher PS masses, larger PS velocities, and denser underlying media would be needed. and Small Magellanic Clouds), the possibility that the PS may lose mass owing to interaction with the parent galaxy is much diminished, compared with the case of a galaxy such as the Milky Way or M 31. In contrast, the opposite may be true in galaxies that are more massive than the Milky Way, with the PSs potentially losing more mass in such massive galaxies (see also Bekki 2011). Therefore, clusters with similar present-day masses in the Milky Way and the Magellanic Clouds are likely to be associated with more massive PSs in the former than in the latter galaxies (but see Chandar et al. 2010). The end result is that the chemical inhomogeneities are expected to be less extreme in Magellanic Cloud clusters of a given (present-day) mass than in Milky Way clusters of similar (present-day) mass 


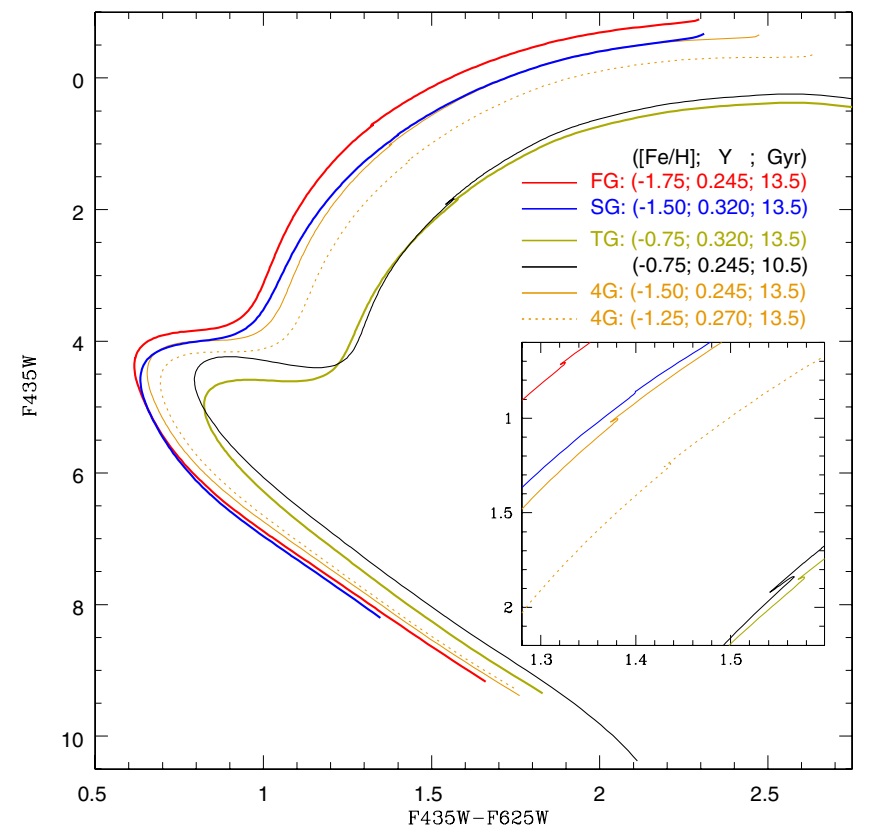

Fig. 6. As in Fig. 2, but for a massive PS after 13 Gyr. PGPUC isochrones represent the different populations presented for this PS: FG (red lines), SG (blue lines), TG (gold lines), and 4G (orange lines). In black lines represents a possible younger TG population. Inner panel is a zoom-in at the RGB bump. This figure is available in color in electronic form.

(see also Mucciarelli et al. 2011a) - and vice-versa in the case of galaxies that are more massive than the Milky Way. This has important potential implications for the interpretation of the integrated light of GCs (e.g., Kaviraj et al. 2007; Rey et al. 2007; Mieske et al. 2008; Georgiev et al. 2009; Coelho et al. 2011; Peacock et al. 2011), potentially leading to parent galaxy massrelated effects.

We note that, according to our scenario, each subsequent stellar generation in $\omega$ Cen need not differ substantially in age. This is in contrast to early claims that $\omega$ Cen harbors stellar populations with an internal age spread that can reach several Gyr (e.g., Hughes \& Wallerstein 2000; Rey et al. 2004; Stanford et al. 2006). We note, however, that these earlier results were obtained without taking into account the possibility of different levels of He enrichment in the different populations, which can lead to biases in the age estimates. In addition, it has also been suggested that the ages of the different populations in $\omega$ Cen are indeed very similar, perhaps to within 2 Gyr (Sollima et al. 2005b; Pancino et al. 2011; D'Antona et al. 2011). That a uniform-age solution is consistent with the cluster's CMD is clearly shown in Fig. 8 of Sollima et al. (2005b), where a fit to high-quality ACS@HST photometry is shown in the constant-age/multiple-Y scenario.

As for the previous PS classes, in panel h of Fig. 5 we show schematically the expected shape of the diagnostic $[\mathrm{Na} / \mathrm{Fe}]$ vs. $[\mathrm{O} / \mathrm{Fe}]$ diagram. The corresponding HR diagrams for this class of PS are shown in Fig. 6.

In terms of the age issue and $\omega$ Cen's CMD, one can observe from Fig. 6 two important points:

- The youngest SGB of the TG population with normal Y is not parallel to the SGB of an older FG population. Coeval SGBs, on the other hand, are parallel. The observations reveal nearly parallel SGBs for the different populations (see, e.g., Fig. 5. of Villanova et al. 2007), and thus imply that there is a small age spread in the cluster.

- The RGB bump of the TG population shows a greater spread in luminosity than the one of the FG population if these populations have the same helium abundances. However, if the TG is helium-rich, the spread in luminosities are predicted to be similar. That the observed spreads are quite similar is supported by Fig. 10 of Rey et al. (2004).

As a final comment regarding the origin of the putative TG (i.e., the more metal-rich) component in $\omega$ Cen, we note that it clearly corresponds to a discrete component in this cluster. However, it is not clear to us how several Gyr can go by after the SN II explosions until such a metal-rich component is formed (see also Gratton et al. 2011). SN Ia do not appear to constitute a feasible solution, since the most metal-rich stars in $\omega$ Cen are also highly enriched in $\mathrm{O}$ and $\mathrm{Na}$ with respect to SG stars (Johnson \& Pilachowski 2010; Marino et al. 2011a; Gratton et al. 2011). Yields of SN II (see Fig. 8 in Limongi \& Chieffi 2003) do show an increase in $\mathrm{Na}$, but not in $\mathrm{O}$ - which means that, according to our scenario, $\mathrm{TG}$ stars will have $[\mathrm{O} / \mathrm{Fe}]$ similar to that of $\mathrm{SG}$ stars, but higher $[\mathrm{Na} / \mathrm{Fe}]$ and $[\mathrm{Fe} / \mathrm{H}]$. This is precisely the behavior that is observed in Fig. 19 of Johnson \& Pilachowski (2010).

According to our scenario, we therefore expect for a very massive progenitor that SG stars are $\mathrm{He}$ - and Na-rich, but O-poor (yields from a combination of pristine gas and massive stars before they explode), with a small enrichment of Fe (provided by a small fraction of FG SN II material). On the other hand, TG stars are expected to be $\mathrm{He}-$, Na-, and Fe-rich, but O-poor. Even though this model predicts separate distributions in the O-Na plane for the FG, SG, and TG stars - a gap that becomes "bridged" when the 4G stars are formed (see Fig. 5, panel h) one should bear in mind that this is only a schematic representation, and the real chemical evolution can be more complex, especially because $4 \mathrm{G}$ stars are formed with material processed by previous generations plus a fraction of pristine gas (if the latter has not been completely expelled). As observed in the O-Na anticorrelation of $\omega$ Cen (Johnson \& Pilachowski 2010; Marino et al. 2010), there is an overlap between metal-poor and metalintermediate branches in the O-Na plane. However, metal-rich stars $([\mathrm{Fe} / \mathrm{H}]>-1.3)$ are all Na-rich, which can also be associated to some degree of He enrichment.

\subsection{General comments}

We presented a scenario for the formation of multiple populations in GCs that makes several non-standard assumptions. We now explain why these assumptions were made.

First, we incorporate into the cluster's evolving chemistry the ejecta of massive stars. Most frequently, however, the contribution of massive stars is ignored, because their winds are very fast (200 $\mathrm{km} \mathrm{s}^{-1} \leq v \leq 2200 \mathrm{~km} \mathrm{~s}^{-1}$; Lamers \& Nugis 2002). We decided to take these winds into account for four main reasons, namely:

- First, massive stars are clearly good candidates to explain populations with high helium abundances without any increase in metals, provided their ejecta is considered before they explode as core-collapse SNe. This is because they go through evolutionary stages during which their atmospheres are comprised entirely of helium (Limongi \& Chieffi 2007; Pastorello et al. 2008; Jeffery \& Hamann 2010, and references therein). Moreover, core-collapse SNe classification divides them into three groups, depending on the elements 
observed in their spectra: i) SNe type II, whose spectra show $\mathrm{H}$ lines; ii) SNe type Ib, whose spectra shows $\mathrm{He}$, but no $\mathrm{H}$ lines; and iii) SNe type Ic, whose spectra show neither $\mathrm{H}$ nor He lines. It has been proposed that these differences are due to the SNe progenitors' different masses, with the progenitors of SNe type II being less massive than those of SNe type $\mathrm{Ib}$, which in turn are less massive than the SNe type Ic progenitors (see, e.g., Leonard 2010, for a recent review). It appears accordingly that the progenitors of SNe type Ib and Ic return to the ISM a large amount of helium before they explode.

- In this sense, the material that arrives at the center of the PS is composed of a mix of pristine gas and the ejecta provided by the winds of massive stars. Before SNe explode, the yields of these winds are similar to the pristine composition early on, but almost completely comprised of He created via hightemperature $\mathrm{H}$ burning towards the end. Naturally, the PS cannot and does not select only the He-enriched wind ejecta to create SG stars; rather, SG stars are formed from a mix of pristine gas (i.e., gas that was not used to form FG stars, plus winds of FG stars) and He-rich winds.

- If PSs have lost a large fraction of their mass in the course of their lives, and their present-day mass is on the order of $1 \%$ of their initial mass only ${ }^{4}$, the escape velocity of the PS was ten times greater than the present-day escape velocity, thus making the retention of the massive stellar winds within the cluster's gravitational potential well much more feasible early on. Moreover, the intracluster gas also plays an important role in decreasing the velocity of the ejected gas. For example, in the case of a GC - a group of stars without intracluster gas - the velocity of the ejected material decreases only because of the action of the GC potential well, and so, if the initial velocity is greater than the escape velocity, that ejecta will be expelled from the GC. However, in the case of a PS - a group of stars with intracluster gas the velocity of the ejecta of stars is decreased by the GC potential well, as well as the interaction with the intracluster gas (e.g., Dopita 1981; Dopita \& Smith 1986; Brown et al. 1995; Kasliwal et al. 2005). This means that, even if the initial velocity of the ejecta is greater than the escape velocity, the ejected gas can still be retained by the PS. This process would naturally increase in importance with the mass of the PS, since more massive PSs have a larger amount of pristine gas that can interact with the ejecta.

- If pristine gas is required to form SG stars (as implied by the Li and F abundances measured in GC stars; see, e.g., Prantzos et al. 2007), the latter must form before the first core-collapse $\mathrm{SNe}$, otherwise the pristine gas has either been expelled or become metal-enriched. Massive star winds are accordingly the only viable source of gas enrichment. Even though one can assume that SG stars will never be enriched in metals, the amount of core-collapse SN explosions required to trigger the formation of these stars might depend on the amount of gas at the center of the PS, which could produce an increase of the metallicity, as in the case of $\omega$ Cen (e.g., Piotto et al. 2005).

We emphasize that stars with extreme He abundances may naturally have extreme abundances of other elements, such as high $\mathrm{Na}$ and low $\mathrm{O}$. Therefore, stars at the high-Na, low-O end of the $\mathrm{O}-\mathrm{Na}$ anticorrelation (panel $\mathrm{h}$ in Fig. 5, and panels $\mathrm{g}$ in

\footnotetext{
4 Decressin et al. (2007a) pointed out that, to reproduce the large fraction of SG stars, it is indeed necessary for the GC to have lost close to $96 \%$ of all FG stars, unless a flat IMF for massive stars is adopted.
}

Figs. 3 and 1) are those that are more naturally expected to have high He abundances. This is similar to the observations of Bragaglia et al. (2010). This would also be qualitatively consistent with the observed correlation between the presence and extent of these abundance anomalies and position along the horizontal branch (e.g., Catelan \& de Freitas Pacheco 1995; Gratton et al. 2010; Marino et al. 2011c). However, to more properly test this scenario, models of massive stars with mass loss for low metallicities and with time-dependent yields are required, but these are unfortunately not yet available in the literature.

Regarding the ratio of FG stars to subsequent generations of stars, the following remarks are in order. If every new generation is formed in the core of the PS, then the number of FG stars decreases more than the number of stars belonging to the subsequent generations, owing to the preferential evaporation of outer stellar members (Decressin et al. 2008). As Carretta et al. (2009b) have suggested, these expelled stars can then become a principal component of the halo field. Moreover, the effect presented by Decressin et al. (2008) can be intensified because of expansion of the PS driven by expulsion of the gas that was not used to form stars (Moeckel \& Bate 2010; Decressin et al. 2010; Trenti et al. 2010). Even though our schematic plots try to represent the effect of the expulsion of FG stars, these figures may to some extent also overestimate the number of FG stars.

Finally, an additional case of PS could in principle exist, that is in-between our definitions of intermediate-mass and lowmass PSs, where only FG and SG stars, but not TG stars, are formed. Such a PS would be expected to be characterized by a large gap in the O-Na diagram, between O/Na-"normal" and $\mathrm{O}$-poor/Na-rich stars. To the best of our knowledge, no such cases have been found in the literature. For such a scenario to materialize, a continuous mechanism responsible for removing the stellar ejecta of super-AGB and AGB stars should be present. This mechanism could be provided by SN Ia explosions; however, a fraction of this gas could still be retained if these explosions are not very close to the PS core, thus allowing the formation of TG stars with a mixed chemical composition.

\section{Quantitative estimates}

We now present a method to estimate the initial mass $M_{\mathrm{I}}$ of the PS that is expected in our scenario, on the basis of the presentday observed ratio of the number of $\mathrm{SG}$ to FG stars $\left(R_{\mathrm{FG}}^{\mathrm{SG}}\right)$.

First, assuming a constant star-formation efficiency $\epsilon$, independent of the chemical composition, the total mass used to form the FG is

$M_{\mathrm{FG}}=M_{\mathrm{I}} \times \epsilon$,

while the remaining pristine gas has a mass of

$M_{\mathrm{g}}=M_{\mathrm{I}} \times(1-\epsilon)$.

The mass used to form the SG stars is then

$M_{\mathrm{SG}}=\left(M_{\mathrm{g}} \times f_{\mathrm{g}}+M_{\mathrm{ej}-\mathrm{FG}}\right) \times \epsilon$,

where $f_{\mathrm{g}}$ and $M_{\mathrm{ej}-\mathrm{FG}}$ are the mass fraction of the pristine gas and the fraction of ejected mass of FG stars that has collapsed to the core of the PS (and thus used up to form SG stars), respectively. We estimate the last term from

$M_{\mathrm{ej}-\mathrm{FG}}=M_{\mathrm{FG}} \times I \times f_{\mathrm{I}}$,

where $I$ is the (time-dependent) fraction of the total mass ejected by massive FG stars, and $f_{\mathrm{I}}$ is the fraction of this ejecta that has 
arrived at the PS core - which we again assume to be entirely used up in the formation of SG stars.

The present-day (i.e., at time $t_{\mathrm{GC}}$ ) number of FG stars is $N_{\mathrm{FG}} \times \eta_{\mathrm{FG}}$, where $N_{\mathrm{FG}}$ is the initial number of FG stars and $\eta_{\mathrm{FG}}$ is the fraction of the $N_{\mathrm{FG}}$ stars that are still alive at time $t_{\mathrm{GC}}$. This can be determined using the $\operatorname{IMF}(\phi)$ from

$\eta_{x}=\int_{0.1}^{x} \phi(m) \mathrm{d} m / \int_{0.1}^{120} \phi(m) \mathrm{d} m$

where $x$ is the highest mass value at time $t_{\mathrm{GC}}$ for the relevant chemical composition. For the maximum stellar mass, we adopted a conservative limit to low metallicities (Stothers \& Chin 1993), while the lowest mass limit was chosen according to Kroupa et al. (1993).

On the other hand, the number of stars that are still bound to the GC after a time $t_{\mathrm{GC}}$ is given by

$N_{\mathrm{FG}-\text { now }}=\eta_{\mathrm{FG}} \times\left(1-S_{\mathrm{L}}\right) \times N_{\mathrm{FG}}$,

where $S_{\mathrm{L}}$ is the fraction of FG stars that have been expelled from the PS by dynamical interactions with the Milky Way and other structures. Moreover, $N_{\mathrm{FG}}$ is related to $M_{\mathrm{FG}}$ with $N_{\mathrm{FG}}=$ $M_{\mathrm{FG}} /\langle m\rangle_{t}$, where $\langle m\rangle_{t}$ is the mean stellar mass, which depends on the time and the chemical composition, except when $t_{\mathrm{GC}}=0$ $\left(\langle m\rangle_{0}\right)$. Accordingly,

$N_{\mathrm{FG}-\text { now }}=\frac{\eta_{\mathrm{FG}} \times\left(1-S_{\mathrm{L}}\right) \times M_{\mathrm{FG}}}{\langle m\rangle_{0}}$.

For the SG, this number is

$N_{\mathrm{SG}-\text { now }}=\frac{\eta_{\mathrm{SG}} \times\left(1-S_{\mathrm{L}}^{\mathrm{SG}}\right) \times M_{\mathrm{SG}}}{\langle m\rangle_{0}}$,

where we have assumed that a fraction $S_{\mathrm{L}}^{\mathrm{SG}}$ of SG stars has also been lost.

Finally, the ratio of $N_{\mathrm{SG}-\text { now }}$ to $N_{\mathrm{FG}-\text { now }}\left(R_{\mathrm{FG}}^{\mathrm{SG}}\right)$, which can be estimated from observations, is

$R_{\mathrm{FG}}^{\mathrm{SG}}=\frac{\eta_{\mathrm{SG}} \times\left[(1-\epsilon) \times f_{\mathrm{g}}+\epsilon \times I \times f_{\mathrm{I}}\right]}{\eta_{\mathrm{FG}} \times\left(1-S_{\mathrm{L}}\right)} \times\left(1-S_{\mathrm{L}}^{\mathrm{SG}}\right)$,

which interestingly does not depend directly on either $M_{\mathrm{I}}$ or $M_{\mathrm{GC}}$, but is related to these quantities indirectly through $S_{\mathrm{L}}$ and $\eta$. In Eq. (9), all variables (including, naturally, $R_{\mathrm{FG}}^{\mathrm{SG}}$ ) can be estimated (by models and/or by observations), except for $S_{\mathrm{L}}$ and $S_{\mathrm{L}}^{\mathrm{SG}}$. Therefore, if a value for $S_{\mathrm{L}}^{\mathrm{SG}}$ is assumed ${ }^{5}, S_{\mathrm{L}}$ can be derived from

$S_{\mathrm{L}}=1-\frac{\eta_{\mathrm{SG}} \times\left[(1-\epsilon) \times f_{\mathrm{g}}+\epsilon \times I \times f_{\mathrm{I}}\right]}{\eta_{\mathrm{FG}} \times R_{\mathrm{FG}}^{\mathrm{SG}}} \times\left(1-S_{\mathrm{L}}^{\mathrm{SG}}\right)$.

To determine the $M_{\mathrm{I}}$ of a PS once $S_{\mathrm{L}}$ has been computed, we note that:

- $M_{\mathrm{I}}$ is related to $M_{\mathrm{FG}}$ and $\epsilon$ through Eq. (1).

\footnotetext{
5 Observations have shown that $\sim 2.5 \%$ of the halo stellar population is $\mathrm{CN}$-strong, thus can be associated with SG stars that once belonged to GCs (Martell \& Grebel 2010). Moreover, the $N$-body simulations of D'Ercole et al. (2008) show that if SG stars are formed in the centers of GCs (corresponding to $\sim 15 \%$ of the total population), after $13 \mathrm{Gyr}$ the fractions of FG and SG stars that are lost are $~ 99 \%$ and $\sim 70 \%$, respectively, with the end result that the GC population is dominated by SG stars ( $80 \%$ are SG stars).
}

- $M_{\mathrm{FG}}$ is related to the present-day mass of FG stars $\left(M_{\mathrm{FG}-\text { now }}\right), S_{\mathrm{L}}$ and the present-day mass fraction of $M_{\mathrm{FG}}$ $\left(\mathfrak{M}_{\mathrm{FG}}\right)$ by $M_{\mathrm{FG}-\text { now }}=M_{\mathrm{FG}} \times\left(1-S_{\mathrm{L}}\right) \times \mathfrak{M}_{\mathrm{FG}}$. The $\mathfrak{M}_{\mathrm{FG}}$ value is determined considering only stars with masses lower than the maximum mass $(x)$ of stars belonging to the FG that are still alive, or

$$
\mathfrak{M}_{x}=\int_{0.1}^{x} m \times \phi(m) \mathrm{d} m / \int_{0.1}^{120} m \times \phi(m) \mathrm{d} m .
$$

- $M_{\mathrm{FG}-\text { now }}$ is related both to $M_{\mathrm{GC}}$ and the fraction of present-day FG stars according to $M_{\mathrm{FG}-\text { now }}=M_{\mathrm{GC}} \times$ $\left(N_{\mathrm{FG}-\text { now }} / N_{\text {now }}\right)$, where $N_{\text {now }}$ is the total present-day number of stars in the $\mathrm{GC}$, or equivalently, $M_{\mathrm{FG}-\text { now }}=$ $M_{\mathrm{GC}} /\left(1+R_{\mathrm{FG}}^{\mathrm{SG}}\right){ }^{6}$

In summary, we find that

$M_{\mathrm{I}}=\frac{M_{\mathrm{GC}}}{\mathfrak{M}_{\mathrm{FG}} \times \epsilon \times\left(1+R_{\mathrm{FG}}^{\mathrm{SG}}\right) \times\left(1-S_{\mathrm{L}}\right)}$,

which relates $M_{\mathrm{I}}$ to $S_{\mathrm{L}}$, which can be estimated from the observations using Eq. (10) (assuming a value for $S_{\mathrm{L}}^{\mathrm{SG}}$ ).

\subsection{The case of NGC 2808}

We now estimate both $S_{\mathrm{L}}$ and $M_{\mathrm{I}}$ for NGC 2808, a GC that we assume to be representative of intermediate-mass PSs.

We use a value of $\epsilon=0.4$ (Parmentier et al. 2008), while the values for $\eta$ can be determined from Eq. (5) if the initial mass of the most massive star of each generation is known; the masses of low-mass stars are assumed not to decrease by a large amount. They are calculated with knowledge of the chemical composition, and the age of each star generation. Here, we assume that both generations have the same age $\left(t_{\mathrm{GC}}=12 \mathrm{Gyr}\right)$, metallicity $(Z=0.001)$, and $\alpha$-element ratio $([\alpha / \mathrm{Fe}]=0.3)$, but different helium abundances $\left(Y_{\mathrm{FG}}=0.245\right.$ and $Y_{\mathrm{SG}}=0.345$; Piotto et al. 2007). This implies maximum stellar masses of $\simeq 0.81 M_{\odot}$ and $\simeq 0.68 M_{\odot}$ for the FG and $\mathrm{SG}$, respectively (Valcarce et al., in prep.). Using a Kroupa et al. (1993) IMF, we also find that $\eta_{\mathrm{FG}}=0.50$ and $\eta_{\mathrm{SG}}=0.44$.

To determine $I$, we use the values from Table 2 of Limongi \& Chieffi (2007). As far as the present study is concerned, the most important conclusions are as follows:

- The first core-collapse SN (initial mass of $120 M_{\odot}$ ) explodes after 3 Myr.

- Only stars more massive than $20 M_{\odot}$ lose a significant amount of mass in this short time interval.

- We obtain a linear relation between the initial mass of the stars and the mass lost after $3 \operatorname{Myr}\left(M_{\text {Lost }}\right.$; see Fig. 7), which reads as follows (with masses given in solar units):

$$
M_{\text {Lost }}(3 \mathrm{Myr})=0.88 \times M_{\text {initial }}-18.25 \text {. }
$$

We assume, for simplicity, that massive stars lose their envelopes at a uniform rate during their lives.

${ }^{6}$ This neglects the mass of the stellar remnants, which constitute $\sim 30 \%$ of the total GC mass after $12 \mathrm{Gyr}$, where the exact remnant mass fraction depends on the detailed dynamical evolution history, metallicity, and the IMF (Kruijssen \& Lamers 2008). On the other hand, the mass-to-light ratio also depends on these same parameters, and varies in the range $M / L_{V} \approx 1$ to $7 M_{\odot} L_{\odot}^{-1}$ at an age of $12 \mathrm{Gyr}$ (Kruijssen \& Lamers 2008). Since the uncertainty in $M / L_{V}$ is much higher than that brought about by neglecting the stellar remnants, we decided to avoid the latter in our first, and admittedly rough, PS mass estimates. 


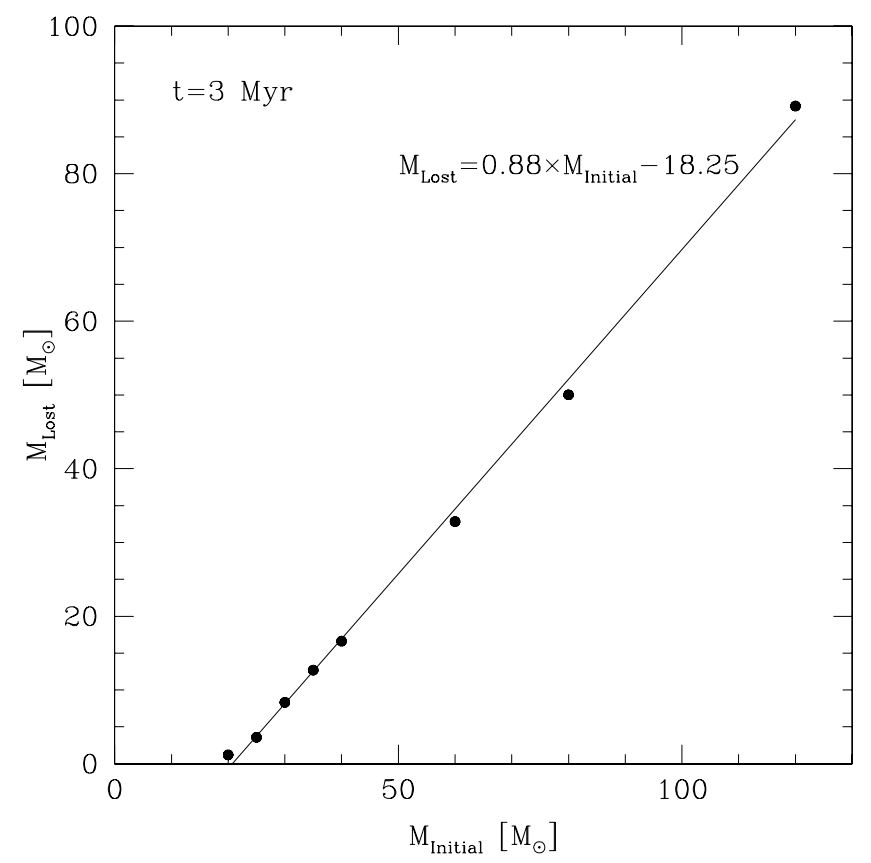

Fig. 7. Relation between the initial mass and the total mass loss by massive stars after 3 Myr. Dots are from Table 2 of Limongi \& Chieffi (2006), where a constant mass loss rate was assumed. The line is our linear fit.

$I$ can thus be estimated as

$I=\int_{20}^{120} M_{\text {Lost }}(m) \times \phi(m) \mathrm{d} m / \int_{0.1}^{120} m \times \phi(m) \mathrm{d} m$,

which at $3 \mathrm{Myr}$ takes on a value of 0.015 . In other words, after 3 Myr stars more massive than $20 M_{\odot}$ will have ejected $1.5 \%$ of the total mass used to form the entire population of stars to which they are associated - low-mass stars included.

Finally, Eq. (10) is reduced to

$S_{\mathrm{L}}=1-\frac{5.28 \times 10^{-1} \times f_{\mathrm{g}}+2.64 \times 10^{-3} \times f_{\mathrm{I}}}{R_{\mathrm{FG}}^{\mathrm{SG}}}$,

which depends on $f_{\mathrm{g}}$ and $f_{\mathrm{I}}$, and where we have assumed, for simplicity, that a negligible number of SG stars has been lost.

Since our model predicts that SG stars should have low $\mathrm{O}$ abundances, to obtain an estimate of $f_{\mathrm{g}}$ we use $[\mathrm{O} / \mathrm{Fe}]_{\min }$ and $[\mathrm{O} / \mathrm{Fe}]_{\max }$, which are the minimum and maximum abundances of this element relative to iron that are observed in the present-day GC. We proceed as follows:

- First, in a cloud of mass $M_{\mathrm{u}}$, the number of particles per unit mass of an element $\mathrm{X}\left(N_{\mathrm{X}}\right)$ is

$N_{\mathrm{X}}=M_{\mathrm{u}} \times F_{\mathrm{X}}$,

where $F_{\mathrm{X}}$ is the mass fraction of element $\mathrm{X}$.

- Then, the ratio of the number of particles per unit mass of $\mathrm{O}$ to Fe in the progenitor cloud of SG stars is

$\left(\frac{N_{\mathrm{O}}}{N_{\mathrm{Fe}}}\right)_{\mathrm{SG}}=\frac{M_{\mathrm{g}} \times f_{\mathrm{g}} \times F_{\mathrm{O}, \mathrm{g}}+M_{\mathrm{FG}} \times I \times f_{\mathrm{I}} \times F_{\mathrm{O}, \mathrm{FG}}}{M_{\mathrm{g}} \times f_{\mathrm{g}} \times F_{\mathrm{Fe}, \mathrm{g}}+M_{\mathrm{FG}} \times I \times f_{\mathrm{I}} \times F_{\mathrm{Fe}, \mathrm{FG}}}$,

where $M_{\mathrm{g}} \times f_{\mathrm{g}}$ and $F_{\mathrm{X}, \mathrm{g}}$ are, respectively, the mass used and the fraction by mass of the element $\mathrm{X}$ in the pristine gas, whereas $M_{\mathrm{FG}} \times I \times f_{\mathrm{I}}$ and $F_{\mathrm{X}, \mathrm{FG}}$ are the mass used and the fraction by mass of the element $\mathrm{X}$ in the mass ejected by FG massive stars.
- Hence, if $F_{\mathrm{Fe}, \mathrm{g}}=F_{\mathrm{Fe}, \mathrm{FG}}=F_{\mathrm{Fe}}$, which means that massive stars do not eject material enriched in $\mathrm{Fe}$ in the first $3 \mathrm{Myr}$, Eq. (17) can be rewritten as

$\left(\frac{N_{\mathrm{O}}}{N_{\mathrm{Fe}}}\right)_{\mathrm{SG}}=\frac{f_{\mathrm{g}} \times(1-\epsilon) \times F_{\mathrm{O}, \mathrm{g}}+\epsilon \times I \times f_{\mathrm{I}} \times F_{\mathrm{O}, \mathrm{FG}}}{\left[f_{\mathrm{g}} \times(1-\epsilon)+\epsilon \times I \times f_{\mathrm{I}}\right] \times \epsilon \times F_{\mathrm{Fe}}}$,

where $M_{\mathrm{g}}$ and $M_{\mathrm{FG}}$ are related to $M_{\mathrm{I}}$ using Eqs. (1) and (2). Relating $F_{\mathrm{O}}$ and $F_{\mathrm{Fe}}$ to $N_{\mathrm{O}}$ and $N_{\mathrm{Fe}}$, respectively (Eq. (16)), Eq. (18) is equivalent to

$\left(\frac{N_{\mathrm{O}}}{N_{\mathrm{Fe}}}\right)_{\mathrm{SG}}=\frac{1}{\epsilon \times(1+c)} \times\left[\left(\frac{N_{\mathrm{O}}}{N_{\mathrm{Fe}}}\right)_{\mathrm{g}}+c\left(\frac{N_{\mathrm{O}}}{N_{\mathrm{Fe}}}\right)_{\mathrm{FG}}\right]$,

where

$c=\frac{\epsilon \times I \times f_{\mathrm{I}}}{(1-\epsilon) \times f_{\mathrm{g}}}$.

- Finally, Eq. (19) can be transformed to relative abundances,

$10^{[\mathrm{O} / \mathrm{Fe}]_{\mathrm{SG}}}=\frac{1}{\epsilon \times(1+c)} \times\left[10^{[\mathrm{O} / \mathrm{Fe}]_{\mathrm{g}}}+c \times 10^{[\mathrm{O} / \mathrm{Fe}]_{\mathrm{FG}}}\right]$,

which means that the ejected gas by FG massive stars must have an $\mathrm{O}$ abundance of

$[\mathrm{O} / \mathrm{Fe}]_{\mathrm{FG}}=\log \left[\frac{\epsilon \times(1+c) \times 10^{[\mathrm{O} / \mathrm{Fe}]_{\mathrm{SG}}}-10^{[\mathrm{O} / \mathrm{Fe}]_{\mathrm{g}}}}{c}\right]$.

We note that the fraction $f_{\mathrm{I}} / f_{\mathrm{g}}$ that is used to compute $c$ is constrained by

$\frac{f_{\mathrm{I}}}{f_{\mathrm{g}}} \geq \frac{1-\epsilon}{\epsilon \times I} \times\left(\frac{10^{\Delta[\mathrm{O} / \mathrm{Fe}]}}{\epsilon}-1\right)$,

where $\Delta[\mathrm{O} / \mathrm{Fe}]=[\mathrm{O} / \mathrm{Fe}]_{\mathrm{g}}-[\mathrm{O} / \mathrm{Fe}]_{\mathrm{SG}}$. Here we have obtained a relation between the fraction $f_{\mathrm{g}}$ of primordial gas used to form SG stars and the fraction $f_{\mathrm{I}}$ of gas ejected by FG stars that are used to form SG stars. We note, by definition, that neither of these values can exceed unity.

One can estimate the ratio in Eq. (23) by assuming that $[\mathrm{O} / \mathrm{Fe}]_{\mathrm{g}}$ and $[\mathrm{O} / \mathrm{Fe}]_{\mathrm{SG}}$ are directly given by $[\mathrm{O} / \mathrm{Fe}]_{\max }$ and $[\mathrm{O} / \mathrm{Fe}]_{\min }$, respectively, as indeed implied by our model. Using the abundance ratios derived by Carretta et al. (2006) in the case of NGC $2808\left([\mathrm{O} / \mathrm{Fe}]_{\max }=0.4\right.$ and $\left.[\mathrm{O} / \mathrm{Fe}]_{\min }=-1.0\right)$, we obtain $f_{\mathrm{I}} \geq 6180 \times f_{\mathrm{g}}$, where, as in Eq. (15), we used $I=0.015$ and $\epsilon=0.4$. This implies that, if SG stars in NGC 2808 were formed using $100 \%$ of the ejected mass by massive FG stars (corresponding to $1.5 \%$ of $M_{\mathrm{I}}$ ), the maximum mass fraction of the primordial gas that can be used to form SG stars must be lower than $0.02 \%$, or else the observed values of $[\mathrm{O} / \mathrm{Fe}]$ cannot be reproduced ${ }^{7}$.

7 Observationally, one finds that the abundances of fragile elements such as $\mathrm{Li}$ and $\mathrm{F}$ are correlated with $\mathrm{O}$ (and anticorrelated with $\mathrm{Na}$; e.g., Pasquini et al. 2005; Smith et al. 2005; Denissenkov et al. 2006; Shen et al. 2010). However, Shen et al. conclude that the slope of the observed Li-O correlation is not what one would expect from simple pollution scenarios, claiming that the polluting gas must be somewhat enriched in $\mathrm{Li}$. If so, and as pointed out by Shen et al., this would rule out massive stars as the main polluters, unless mechanisms can be found through which these massive stars can produce Li. In contrast to their conclusions, however, the chemical evolution models of Lind et al. (2011) account very well for the observed $\mathrm{Li}$ abundances in NGC 6397, when we assume that massive stars of mass between 20 and 


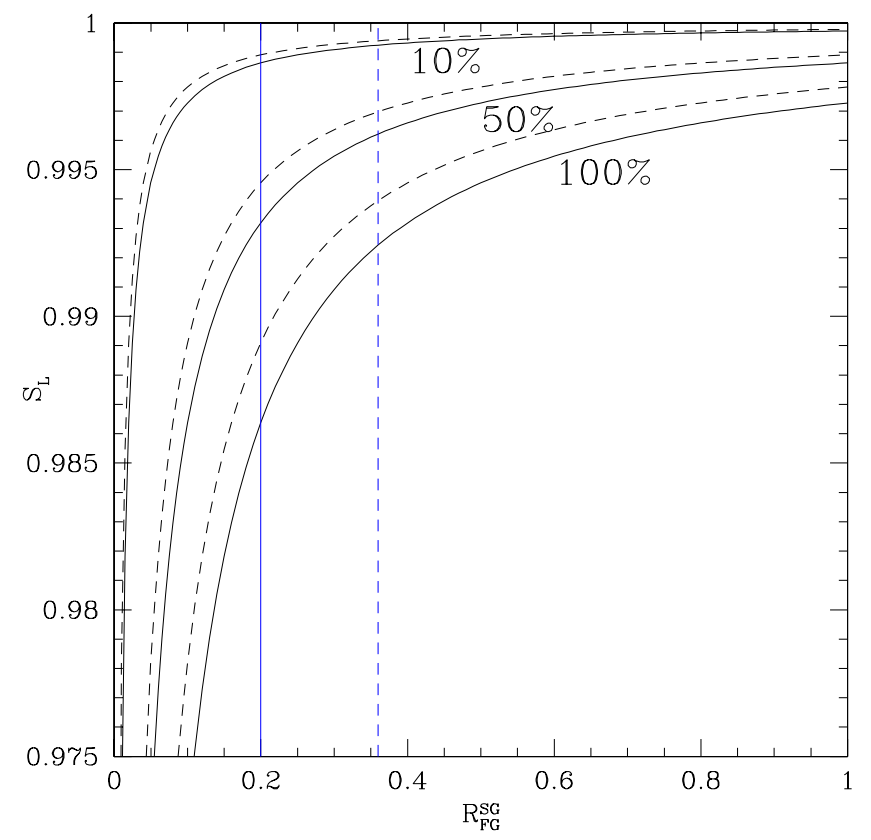

Fig. 8. Fraction of FG stars that an intermediate-mass PS must lose $\left(S_{\mathrm{L}}\right)$ to reproduce the present-day ratio of the number of SG to FG stars. Black curves represent different mass fractions of gas ejected by massive FG stars in $3 \mathrm{Myr}$ (values of $f_{\mathrm{I}}$ in percentages) used to form the SG stars when $\epsilon$ is $40 \%$ and when $S_{\mathrm{L}}^{\mathrm{SG}}$ is negligible (solid lines) or $20 \%$ (dashed lines). The vertical lines represent the observed ratio of SG to FG stars $\left(R_{\mathrm{FG}}^{\mathrm{SG}}\right)$ estimated from the number of red and blue MS stars (solid line, from Piotto et al. 2007) and from the O-Na anticorrelation (dashed line, from Carretta et al. 2009b), as observed in the case of NGC 2808.

Even though the derived limit for $f_{\mathrm{g}}$ implies that $\mathrm{O}$ is completely depleted in the gas ejected by massive FG stars $^{8}$, it can be used to constrain the minimum value of $S_{\mathrm{L}}$ depending on $f_{\mathrm{g}}$ (or $f_{\mathrm{I}}$ ). Then, using Eq. (15), the fraction of FG stars that have been expelled is

$S_{\mathrm{L}} \geq 1-2.73 \times 10^{-3} \times f_{\mathrm{I}} / R_{\mathrm{FG}}^{\mathrm{SG}}$.

Figure 8 shows the minimum fraction of FG stars that NGC 2808 has lost, according to our model. In the specific case of this GC, $R_{\mathrm{FG}}^{\mathrm{SG}}$ can be determined by two methods:

- Using the MS split (Piotto et al. 2007), where 63\% of the population is in the reddest MS, while $13 \%$ is in the bluest MS, and both populations can be associated with FG and $\mathrm{SG}$ stars, respectively - thus implying an $R_{\mathrm{FG}}^{\mathrm{SG}}(\mathrm{MS}) \approx 0.20$.

$120 M_{\odot}$ are the polluters - provided that their ejecta are suitably diluted with pristine material. Clearly, the chemical evolution of $\mathrm{Li}$ is far from being straightforward, as the remarkably constant Li abundance with metallicity observed by Monaco et al. (2010) in $\omega$ Cen also shows. In addition, it is important to bear in mind that it is not straightforward to interpret Li abundance observations, given the many ill-constrained physical mechanisms that may play a relevant role in defining surface Li abundances in present-day stars (e.g., Mucciarelli et al. 2011b).

${ }^{8}$ Massive stars do indeed produce oxygen, albeit to a lower extent than they produce iron. However, the material chemically enriched in elements heavier than $\mathrm{C}, \mathrm{He}$, and $\mathrm{H}$, in the case of the progenitors of $\mathrm{SNe}$ type Ic, type Ib, and type II, respectively, is not expelled via winds, but rather as $\mathrm{SNe}$ ejecta. This means that only PSs that can retain the ejecta of $\mathrm{SNe}$ can also have an enrichment in these heavier elements. This is observed in the O-Na anticorrelation of $\omega \mathrm{Cen}$, when abundance data are separated into bins of metallicity (Johnson \& Pilachowski 2010; Marino et al. 2011a).
However, this method gives the minimum value for $R_{\mathrm{FG}}^{\mathrm{SG}}$ because, according to our scenario, an unknown fraction of the stars in the redder MS were formed after some tens of Myr from material processed by super-AGB or AGB stars (thus belonging to later stellar generations; see Fig. 3). These young stars in the redder MS must have chemical compositions (and in particular helium abundances) similar to those of FG stars, but still displaying abundance variations in some of the light elements.

- Using the O-Na anticorrelation, where stars with low $\mathrm{Na}$ abundances belong to the FG and stars with low $\mathrm{O}$ abundances belong to the SG, whereas stars that belong to neither of these groups are TG stars. Following the definitions of Carretta et al. (2009b) for primordial, intermediate, and extreme populations, $R_{\mathrm{FG}}^{\mathrm{SG}}$ can be determined by dividing the fractions of "extreme" stars by the fraction of "primordial" stars. This gives an $R_{\mathrm{FG}}^{\mathrm{SG}}(\mathrm{O}-\mathrm{Na})=0.36$.

In Fig. 8, these $R_{\mathrm{FG}}^{\mathrm{SG}}$ ratios are represented by blue vertical lines, with the solid line corresponding to the MS CMD constraint, and the dashed one to the O-Na diagram constraint. If SG stars were formed using the total mass of massive FG stars $\left(f_{\mathrm{I}}=100 \%\right)$, the minimum $S_{\mathrm{L}} \sim 0.992$ for $R_{\mathrm{FG}}^{\mathrm{SG}}(\mathrm{O}-\mathrm{Na})$, while for $R_{\mathrm{FG}}^{\mathrm{SG}}(\mathrm{MS})$ the minimum $S_{\mathrm{L}} \sim 0.986$. However, if $f_{\mathrm{I}}=10 \%$, the minimum $S_{\mathrm{L}}$ values are $\sim 0.999$ and $\sim 0.998$ for $R_{\mathrm{FG}}^{\mathrm{SG}}(\mathrm{O}-\mathrm{Na})$ and $R_{\mathrm{FG}}^{\mathrm{SG}}(\mathrm{MS})$, respectively. Even though these values are large, the initial-tofinal mass ratio of GCs depends on the orbit of each PS around the Milky Way, which can completely destroy them if the interaction is strong enough (e.g., Kruijssen et al. 2011).

Using the range of $S_{\mathrm{L}}$ values determined with $f_{\mathrm{I}}=1$ and Eq. (12), the minimum $M_{\mathrm{I}}$ of NGC 2808 is estimated to be between 3.8 and $5.9 \times 10^{8} M_{\odot}$, where we used $\mathfrak{M}_{0.81}=0.504 .^{9}$ The large fraction of cluster mass lost makes our scenario more plausible, because the escape velocity of the PS of NGC 2808 was between $\sim 860$ and $\sim 1060 \mathrm{~km} \mathrm{~s}^{-1}$ (and possibly higher, depending on how many SG stars are lost), instead of the present-day $\sim 50 \mathrm{~km} \mathrm{~s}^{-1}$ (Gnedin et al. 2002). Unfortunately, there are no estimates of the $M_{\mathrm{I}}$ for NGC 2808 in the literature. However, the orbit of this GC suggests that its PS was much more massive than the present-day GC, owing to interaction with the Milky Way (Casetti-Dinescu et al. 2007).

Another important point that should be emphasized is that our scenario naturally explains the discrete nature of the stellar populations observed in NGC 2808. This is because, after the first core-collapse SN explosion and the triggered formation of SG stars, there will be a period of time without star formation, because of the effects of subsequent FG core-collapse $\mathrm{SNe}$, which are interspersed with SG core-collapse SNe, and then followed by SNe type Ia. One should expect that this process is repeated every time that a new generation is born, but every time with a chemical composition more similar to the FG. Accordingly, the observational detection of the differences between subsequent populations (TG in this case) becomes increasingly more difficult.

\subsection{The case of $\omega$ Cen}

As usual, the case of $\omega$ Cen is more complex than for other GCs, because in both its CMD and spectroscopic analyses alike

9 This assumes that SG stars have not been lost during the PS's evolution. If, on the other hand, $70 \%$ of SG stars have been expelled from the PS, as suggested by D'Ercole et al. (2008), NGC 2808's minimum $M_{\mathrm{I}}$ values are increased, leading to masses between 1.3 and $2.0 \times 10^{9} M_{\odot}$. 


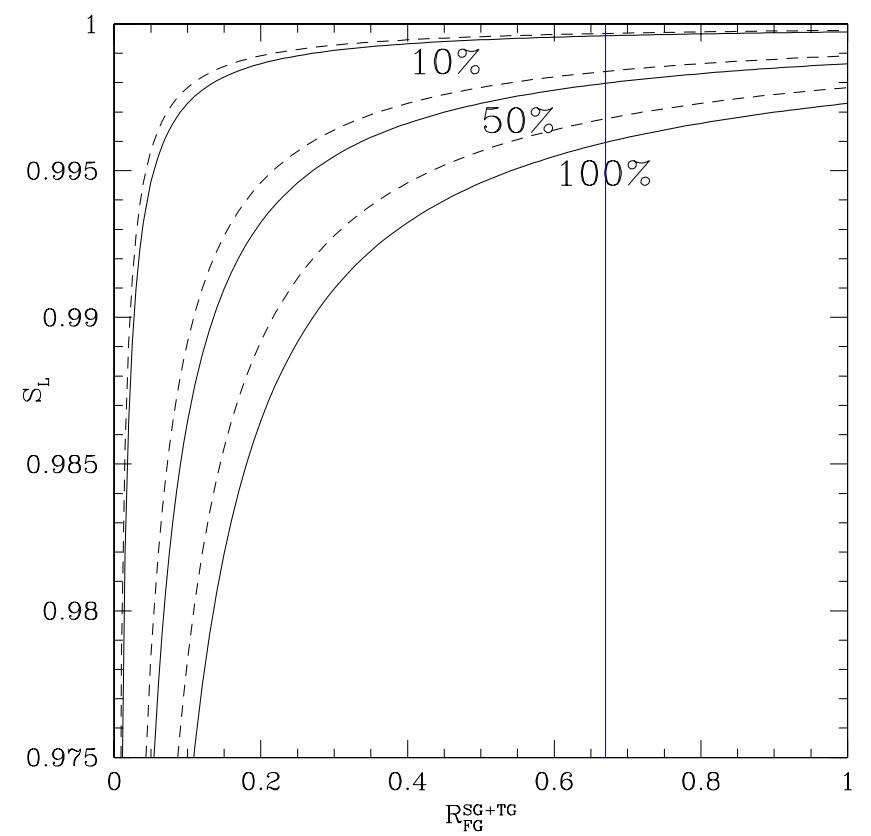

Fig. 9. As in Fig. 8, but in the case of $\omega$ Cen. The vertical line represents $R_{\mathrm{FG}}^{\mathrm{SG}+\mathrm{TG}}=0.67$ (see text).

a large number of populations is observed (Sect. 1). However, we used the ratios of the different populations given in Table 4 of Villanova et al. (2007) to estimate the initial mass of this GC, as required to form SG and TG stars from gas previously processed by FG massive stars (plus a fraction of pristine gas).

In the formation scenario for massive PSs, which is the case assumed for $\omega$ Cen, there is only a small difference in metallicity between FG and SG stars, but a sizeable difference in the helium abundance. As for NGC 2808, we associate a large fraction of the reddest MS of the cluster with FG stars (57\%), while SG stars are associated with the bluest MS (33\%). However, in this scenario the gas ejected by massive stars that is not used to form SG stars is used to form TG stars - which, according to our scenario, will be more metal-enhanced than the SG. We associate these putative TG stars with the MS-a component (containing 5\% of all MS stars) of Villanova et al. (2007), which was proposed to be related to the cluster's most metal-rich RGB sequence (RGB-a in Sollima et al. 2005a). With this in mind, we rewrite Eq. (9) as

$R_{\mathrm{FG}}^{\mathrm{SG}+\mathrm{TG}}=\frac{N_{\mathrm{SG}-\text { now }}+N_{\mathrm{TG}-\text { now }}}{N_{\mathrm{FG}}}$.

In Sect. 4.1, we estimated a value for $I=0.015$, which might have been overestimated. In the case of $\omega$ Cen, we again use the same value, though in this case it could instead be an underestimate, because after 3 Myr massive FG stars continue to return gas to the medium that is used to form SG and TG stars. We also use the same value of $\eta_{\mathrm{FG}}$ estimated for NGC 2808, and assume that $\eta_{\mathrm{SG}+\mathrm{TG}} \approx \eta_{\mathrm{SG}}\left(m_{\mathrm{max}-\mathrm{SG}} \approx 0.68 M_{\odot}\right)$, owing to the small percentage of TG stars. Finally, using Eq. (23), we obtain $f_{\mathrm{I}} \geq 7800 f_{\mathrm{g}}$, which determines a minimum value of $S_{\mathrm{L}}$ for $\omega$ Cen of

$S_{\mathrm{L}} \geq 1-2.71 \times 10^{-3} \times f_{\mathrm{I}} / R_{\mathrm{FG}}^{\mathrm{SG}+\mathrm{TG}}$.

In Fig. 9, we show the relation between $S_{\mathrm{L}}$ and $R_{\mathrm{FG}}^{\mathrm{SG}+\mathrm{TG}}$, where the blue vertical line corresponds to $R_{\mathrm{FG}}^{\mathrm{SG}+\mathrm{TG}}=0.67$, as obtained from the Villanova et al. (2007) number counts. In the case where $f_{\mathrm{I}}=1$, the fraction of FG stars expelled must be higher than $99.6 \%$ of the initial number, implying a $M_{\mathrm{I}} \approx 2.2 \times$ $10^{9} M_{\odot}-$ which is between $\approx 4$ and 17 times higher than the estimates obtained on the basis of $N$-body simulations (e.g., Bekki \& Freeman 2003; Mizutani et al. 2003; Wylie-de Boer \& Cottrell 2009 , with favored $M_{\mathrm{I}}$ values of $1.3,5.8$, and $5.0 \times 10^{8} M_{\odot}$, respectively $)^{10}$. While our estimates of $M_{\mathrm{I}}$ thus appear higher than the most likely (dynamical) value for this PS, we must stress that more detailed computations, incorporating the ingredients described in the next subsection, are clearly required before we are in a position to provide more than only a rough estimate of a PS's $M_{\mathrm{I}}$ value.

\subsection{Required improvements}

In previous sections, we related two GCs to a massive PS ( $\omega$ Cen) and to an intermediate-mass PS (NGC 2808). However, we did not relate any GC to low-mass PSs, owing to the difficulty pointed out in Sect. 2.2, namely the treatment of type Ia SNe. To solve this problem, models of GC evolution are needed that must take properly into account: i) $\mathrm{SNe}$ explosions taking place at different positions inside the GCs; ii) interactions between gases; and iii) the fraction of binary systems that are possible SN type Ia progenitors.

In general, a more precise determination of the following parameters is also required:

- $\epsilon$ : the observed values of this parameter vary between 0.2 and 0.4 (Parmentier et al. 2008), which affects estimates of $M_{\mathrm{I}}$, higher values implying more mass ejected by massive FG stars, which can accordingly be used to form more SG stars.

- IMF: as shown by (e.g.) Skillman (2008), for very low metallicities the IMF predicts more massive stars. However, a proper evaluation of IMFs for metallicities similar to those observed in GCs is required (e.g., Sollima et al. 2007), including the case of high-helium environments.

- I: more robust estimates of this parameter require evolutionary tracks for massive stars with mass loss for low metallicities, along with their yields (e.g., Herwig 2004, for intermediate-mass stars). Moreover, since the properties of these winds depend strongly on the metallicity (lowmetallicity stars releasing a smaller amount of mass), it is possible that a trend between subsequent generations and the metallicity exists. However, it is impossible at present to estimate how sharp this trend is, since metallicity also changes the other two important parameters used in our approach: the IMF and $\epsilon$ (e.g., Skillman 2008; Dib et al. 2011).

- Other ingredients: there are many other possible mechanims that may play an important role in the formation of multiple populations with high helium abundances, but which we did not consider in our analysis. These include, e.g., massive binary stars (De Mink et al. 2009), stellar collisions (Sills \& Glebbeek 2010), and "localized enrichment" (Marcolini et al. 2009), among others (see also Parmentier 2004; Smith 2006, 2010; and de Grijs 2010 and Martell 2011, for recent reviews). Further work incorporating these potentially important ingredients is strongly encouraged.

Finally, a solid model for the evolution of GCs and their multiple populations must consider the evolution of the PS structure

10 Here too we have assumed that SG stars have not been lost during the PS's evolution. If $70 \%$ of SG stars have been expelled from the PS (D'Ercole et al. 2008), $\omega$ Cen's $M_{\mathrm{I}}$ value is increased to $7.4 \times 10^{9} M_{\odot}$, leading to overestimates of $M_{\mathrm{I}}$ by factors in the range between $\approx 13$ and 57 with respect to the aforementioned $N$-body studies. 
after the expulsion of pristine gas, which leads to an expansion of the PS and a period of subsequent star loss (Moeckel \& Bate 2010). Moreover, in a model where massive stars are considered to play an important role in the evolution of GCs, the formation of low-mass stars cannot be assumed to be instantaneous, because proto-low-mass stars have contraction times of several tens of Myr (Bernasconi \& Maeder 1996), thus could become contaminated in that period of time by the winds of massive stars (Newsham \& Terndrup 2007; Tsujimoto et al. 2007).

\section{Summary}

We have provided an overview of some of the most recent scenarios developed to explain the formation of multiple populations in GCs. We have also presented a new scenario, whose chief difference from previous ones is the classification of GCs by their initial mass: i) the most massive PSs can retain the ejecta of massive stars (winds and core-collapse SN ejecta); ii) intermediate-mass PSs can only retain the winds of massive stars, but not the SNe ejecta; and iii) low-mass PSs can only retain the slow winds of intermediate-mass stars (see also Bekki 2011).

To avoid the classical argument against the possibility of using the ejecta of massive stars because of the high velocity of their winds (from hundreds to some thousands of $\mathrm{km} \mathrm{s}^{-1}$ ), in our scenario we postulate that: i) GCs were (much) more massive in the past, hence had escape velocities of some hundreds of $\mathrm{km} \mathrm{s}^{-1}$, and a large percentage of FG stars and the pristine gas were expelled by core-collapse $\mathrm{SNe}$ and/or SNe type Ia, and the interaction with their host galaxy; and ii) after the formation of the FG of stars, the remaining pristine gas begins to fall to the center of the PS, interacting with massive stellar winds that try to escape the PS. An important aspect of our scenario is that it can naturally explain the discrete nature of FG and (especially) SG stellar populations, as observed in GCs.

In general, it is exceedingly difficult to estimate the PS mass $M_{\mathrm{I}}$ directly from the GC's present-day mass $M_{\mathrm{GC}}$, because $M_{\mathrm{GC}}$ depends on the GC's past dynamical history, including interactions with the Galaxy. On the other hand, our scenario allows order-of-magnitude estimates from different empirical estimates of the present-day number ratio of present-day FG to subsequent stellar generations that may be present in individual GCs. This leads to the minimum $M_{\mathrm{I}}$ values for NGC 2808 in the range between about 3.8 and $5.9 \times 10^{8} M_{\odot}$, whereas for $\omega$ Cen we obtain $2.2 \times 10^{9} M_{\odot}$. In the case of NGC 2808, no previous $M_{\mathrm{I}}$ estimates are available, but its orbit is indicative of a massive progenitor (Casetti-Dinescu et al. 2007). In the case of $\omega$ Cen, the mass implied by our scenario is higher than that inferred by $N$-body simulations (Bekki \& Freeman 2003; Mizutani et al. 2003; Wylie-de Boer \& Cottrell 2009). Further work is clearly required, before we are in a position to provide more than a rough estimate of a PS's $M_{\mathrm{I}}$ value, based on present-day observables.

Our scenario can also explain why the O-Na anticorrelation does not follow the same pattern for all GCs, where extreme high-Na/low-O stars are SG stars formed with material processed by massive stars and a fraction of the pristine gas (massive and intermediate-mass PSs), while normal high-Na/low-O ones are SG stars formed with material ejected by super-AGB or AGB stars (low-mass PSs). Subsequent generations are formed with a mixture of material from pre-existing generations, where the last stars to form (i.e., the youngest stars) are more chemically similar to FG stars.
Naturally, we are well aware that our scenario represents but a first "toy model" that will require extensive improvements, including three-dimensional hydrodynamical modeling, before it can provide more detailed, quantitative predictions about the nature and properties of the multiple populations that are now commonly observed in GCs. In this sense, detailed numerical investigations, such as the one carried out by Bekki (2011), are strongly encouraged.

Acknowledgements. We warmly thank Allen V. Sweigart for many helpful comments and discussions throughout the course of this project. Useful discussions with Alvio Renzini, Santi Cassisi, Alejandro Clocchiatti, Andreas Reisenegger, and Manuela Zoccali are gratefully acknowledged. We are also grateful to the anonymous referee, whose comments and suggestions have helped improve the presentation of our results. Support for A.A.R.V. and M.C. is provided by the Ministry for the Economy, Development, and Tourism's Programa Inicativa Científica Milenio through grant P07-021-F, awarded to The Milky Way Millennium Nucleus; by Proyecto Basal PFB-06/2007; by FONDAP Centro de Astrofísica 15010003; and by Proyecto FONDECYT Regular \#1110326. A.A.R.V. acknowledges additional support from Proyecto ALMA-Conicyt 31090002, MECESUP2, and SOCHIAS.

\section{References}

Altmann, M., Catelan, M., \& Zoccali, M. 2005, A\&A, 439, L5 Bedin, L. R., Piotto, G., Anderson, J., et al. 2004, ApJ, 605, L125 Bekki, K. 2011, MNRAS, 412, 2241

Bekki, K., \& Freeman, K. C. 2003, MNRAS, 346, L11

Bekki, K., \& Norris, J. E. 2006, ApJ, 637, L109

Bellini, A., Piotto, G., Bedin, L. R., et al. 2009, A\&A, 507, 1393

Bellini, A., Bedin, L. R., Piotto, G., et al. 2010, AJ, 140, 631

Bernasconi, P. A., \& Maeder, A. 1996, A\&A, 307, 829

Bragaglia, A., Carretta, E., Gratton, R. G., et al. 2010, ApJ, 720, L41

Brown, J. H., Burkert, A., \& Truran, J. W. 1991, ApJ, 376, 115

Brown, J. H., Burkert, A., \& Truran, J. W. 1995, ApJ, 440, 666

Bruzual, G., \& Charlot, S. 2003, MNRAS, 344, 1000

Calamida, A., Bono, G., Stetson, P. B., et al. 2009, ApJ, 706, 1277

Calamida, A., Bono, G., Stetson, P. B., et al. 2010, Mem. Soc. Astron. Ital. Suppl., 14, 95

Caloi, V., \& D'Antona, F. 2011, MNRAS, accepted [arXiv: 1106.0810]

Cannon, R. D., \& Stobie, R. S. 1973, MNRAS, 162, 207

Carretta, E., Bragaglia, A., Gratton, R. G., et al. 2006, A\&A, 450, 523

Carretta, E., Bragaglia, A., Gratton, R., \& Lucatello, S. 2009a, A\&A, 505, 139

Carretta, E., Bragaglia, A., Gratton, R. G., et al. 2009b, A\&A, 505, 117

Carretta, E., Bragaglia, A., Gratton, R. G., et al. 2010a, A\&A, 516, A55

Carretta, E., Gratton, R. G., Lucatello, S., et al. 2010b, ApJ, 722, 1

Carretta, E., Lucatello, S., Gratton, R., Bragaglia, A., \& D’Orazi, V. 2011, A\&A, 533, A69

Casetti-Dinescu, D. I., Girard, T. M., Herrera, D., et al. 2007, AJ, 134, 195

Cassisi, S., Salaris, M., Pietrinferni, A., et al. 2008, ApJ, 672, L115

Cassisi, S., Salaris, M., Anderson, J., et al. 2009, ApJ, 702, 1530

Catelan, M., \& de Freitas Pacheco, J. A. 1995, A\&A, 297, 345

Chandar, R., Fall, S. M., \& Whitmore, B. C. 2010, ApJ, 711, 1263

Coelho, P., Percival, S. M., \& Salaris, M. 2011, ApJ, 734, 72

Cohen, J. G., Kirby, E. N., Simon, J. D., \& Geha, M. 2010, ApJ, 725, 288

Conroy, C. 2011, ApJ, submitted [arXiv: 1101.2208]

Conroy, C., \& Spergel, D. N. 2011, ApJ, 726, 36

Da Costa, G. S., Held, E. V., Saviane, I., \& Gullieuszik, M. 2009, ApJ, 705, 1481

Dalessandro, E., Salaris, M., Ferraro, F. R., et al. 2011, MNRAS, 410, 694

D'Antona, F., Gratton, R., \& Chieffi, A. 1983, Mem. Soc. Astron. Italiana, 54, 173

D’Antona, F., Caloi, V., Montalbán, J., Ventura, P., \& Gratton, R. 2002, A\&A, 395,69

D’Antona, F., Bellazzini, M., Caloi, V., et al. 2005, ApJ, 631, 868

D'Antona, F., Ventura, P., \& Caloi, V. 2007, in From Stars to Galaxies: Building the Pieces to Build Up the Universe, ed. A. Vallenari, R. Tantalo, L. Portinari, \& A. Moretti, ASP Conf. Ser., 374, 155

D'Antona, F., Ventura, P., Caloi, V., et al. 2010, ApJ, 715, L63

D'Antona, F., D'Ercole, A., Marino, A. F., et al. 2011, ApJ, 736, 5

D'Cruz, N. L., Dorman, B., Rood, R. T., \& O'Connell, R. W. 1996, ApJ, 466, 359

D’Cruz, N. L., O’Connell, R. W., Rood, R. T., et al. 2000, ApJ, 530, 352

de Grijs, R. 2010, Roy. Soc. Lond. Phil. Trans. Ser. A, 368, 693

De Mink, S. E., Pols, O. R., Langer, N., \& Izzard, R. G. 2009, A\&A, 507, L1

Decressin, T., Charbonnel, C., \& Meynet, G. 2007a, A\&A, 475, 859 
Decressin, T., Meynet, G., Charbonnel, C., Prantzos, N., \& Ekström, S. 2007b, A\&A, 464, 1029

Decressin, T., Baumgardt, H., \& Kroupa, P. 2008, A\&A, 492, 101

Decressin, T., Baumgardt, H., Charbonnel, C., \& Kroupa, P. 2010, A\&A, 516, A73

Denissenkov, P. A., Pinsonneault, M., \& Terndrup, D. M. 2006, ApJ, 651, 438

D’Ercole, A., Vesperini, E., D’Antona, F., McMillan, S. L. W., \& Recchi, S. 2008, MNRAS, 391, 825

D’Ercole, A., D’Antona, F., Ventura, P., Vesperini, E., \& McMillan, S. L. W. 2010, MNRAS, 407, 854

D’Ercole, A., D'Antona, F., \& Vesperini, E. 2011, MNRAS, 736

Di Criscienzo, M., Ventura, P., D’Antona, F., Milone, A., \& Piotto, G. 2010, MNRAS, 408, 999

Di Criscienzo, M., D’Antona, F., Milone, A. P., et al. 2011, MNRAS, 791

Di Fazio, A. 1986, Mem. Soc. Astron. Italiana, 57, 465

Di Fazio, A., \& Renzini, A. 1980, in Star Formation, ed. J. E. Hesser, IAU Symp., 85,417

Dib, S., Piau, L., Mohanty, S., \& Braine, J. 2011, MNRAS, 891

Dinescu, D. I. 2002, in Omega Centauri, A Unique Window into Astrophysics, ed. F. van Leeuwen, J. D. Hughes, \& G. Piotto, ASP Conf. Ser., 265, 365

Dopita, M. A. 1981, ApJ, 246, 65

Dopita, M. A., \& Smith, G. H. 1986, ApJ, 304, 283

Fall, S. M., \& Zhang, Q. 2001, ApJ, 561, 751

Ferraro, F. R., Dalessandro, E., Mucciarelli, A., et al. 2009, Nature, 462, 483

Freyhammer, L. M., Monelli, M., Bono, G., et al. 2005, ApJ, 623, 860

Georgiev, I. Y., Hilker, M., Puzia, T. H., Goudfrooij, P., \& Baumgardt, H. 2009, MNRAS, 396, 1075

Geyer, E. H. 1967, ZAp, 66, 16

Gnedin, O. Y., Zhao, H., Pringle, J. E., et al. 2002, ApJ, 568, L23

Gratton, R., Sneden, C., \& Carretta, E. 2004, ARA\&A, 42, 385

Gratton, R. G., Carretta, E., Bragaglia, A., Lucatello, S., \& D’Orazi, V. 2010, A\&A, 517, A81

Gratton, R., Johnson, C. I., Lucatello, S., D’Orazi, V., \& Pilachowski, C. A. 2011, A\&A, in press [arXiv: 1105. 5544]

Harris, W. E. 1996, AJ, 112, 1487

Herwig, F. 2004, ApJS, 155, 651

Hilker, M., Kayser, A., Richtler, T., \& Willemsen, P. 2004, A\&A, 422, L9

Hughes, J., \& Wallerstein, G. 2000, AJ, 119, 1225

Jeffery, C. S., \& Hamann, W. 2010, MNRAS, 404, 1698

Johnson, C. I., \& Pilachowski, C. A. 2010, ApJ, 722, 1373

Johnson, C. I., Pilachowski, C. A., Michael Rich, R., \& Fulbright, J. P. 2009, ApJ, 698, 2048

Kalirai, J. S., Saul Davis, D., Richer, H. B., et al. 2009, ApJ, 705, 408

Kasliwal, M. M., Lovelace, R. V. E., \& Houck, J. R. 2005, ApJ, 630, 875

Kaviraj, S., Sohn, S. T., O'Connell, R. W., et al. 2007, MNRAS, 377, 987

Komiya, Y., Habe, A., Suda, T., \& Fujimoto, M. Y. 2010, ApJ, 717, 542

Kroupa, P., Tout, C. A., \& Gilmore, G. 1993, MNRAS, 262, 545

Kruijssen, J. M. D., \& Lamers, H. J. G. L. M. 2008, A\&A, 490, 151

Kruijssen, J. M. D., Pelupessy, F. I., Lamers, H. J. G. L. M., Portegies Zwart,

S. F., \& Icke, V. 2011, MNRAS, 398

Lamers, H. J. G. L. M., \& Nugis, T. 2002, A\&A, 395, L1

Lehnert, M. D., Bell, R. A., \& Cohen, J. G. 1991, ApJ, 367, 514

Leonard, D. C. 2010, Ap\&SS, in press

Limongi, M., \& Chieffi, A. 2003, ApJ, 592, 404

Limongi, M., \& Chieffi, A. 2006, ApJ, 647, 483

Limongi, M., \& Chieffi, A. 2007, in The Multicolored Landscape of Compact Objects and Their Explosive Origins, ed. T. di Salvo, G. L. Israel, L. Piersant, et al., AIP Conf. Ser., 924, 226

Lind, K., Charbonnel, C., Decressin, T., et al. 2011, A\&A, 527, A148

Marcolini, A., Gibson, B. K., Karakas, A. I., \& Sánchez-Blázquez, P. 2009, MNRAS, 395, 719

Marino, A. F., Milone, A. P., Piotto, G., Villanova, S., Bedin, L. R., Bellini, A., \& Renzini, A. 2009, A\&A, 505, 1099

Marino, A. F., Piotto, G., Gratton, R., et al. 2010, in IAU Symp. 268, ed. C. Charbonnel, M. Tosi, F. Primas, \& C. Chiappini, 183

Marino, A. F., Milone, A. P., Piotto, G., et al. 2011a, ApJ, 731, 64

Marino, A. F., Sneden, C., Kraft, R. P., et al. 2011b, A\&A, 532, A8

Marino, A. F., Villanova, S., Milone, A. P., et al. 2011c, ApJ, 730, L16

Martell, S. L. 2011, Astron. Nachr., 332, 467

Martell, S. L., \& Grebel, E. K. 2010, A\&A, 519, A14

Matsunami, N., Obi, S., Shimoda, M., Takase, B., \& Takebe, H. 1959, PASJ, 11, 9

McLaughlin, D. E., \& Fall, S. M. 2008, ApJ, 679, 1272

Mieske, S., Hilker, M., Bomans, D. J., et al. 2008, A\&A, 489, 1023

Milone, A. P., Bedin, L. R., Piotto, G., et al. 2008, ApJ, 673, 241

Mizutani, A., Chiba, M., \& Sakamoto, T. 2003, ApJ, 589, L89
Moeckel, N., \& Bate, M. R. 2010, MNRAS, 404, 721

Monaco, L., Bonifacio, P., Sbordone, L., Villanova, S., \& Pancino, E. 2010, A\&A, 519, L3

Mucciarelli, A., Cristallo, S., Brocato, E., et al. 2011a, MNRAS, 413, 837

Mucciarelli, A., Salaris, M., Lovisi, L., et al. 2011b, MNRAS, 412, 81

Murray, S. D., \& Lin, D. N. C. 1992, ApJ, 400, 265

Murray, S. D., \& Lin, D. N. C. 1993, in The Globular Cluster-Galaxy Connection, ed. G. H. Smith, \& J. P. Brodie, ASP Conf. Ser., 48, 738

Nakasato, N., Mori, M., \& Nomoto, K. 2000, ApJ, 535, 776

Nataf, D. M., Gould, A., Pinsonneault, M. H., \& Stetson, P. B. 2011, ApJ, 736, 94

Newsham, G., \& Terndrup, D. M. 2007, ApJ, 664, 332

Norris, J. E. 2004, ApJ, 612, L25

Origlia, L., Rich, R. M., Ferraro, F. R., et al. 2011, ApJ, 726, L20

Pancino, E., Seleznev, A., Ferraro, F. R., Bellazzini, M., \& Piotto, G. 2003, MNRAS, 345, 683

Pancino, E., Mucciarelli, A., Sbordone, L., et al. 2011, A\&A, 527, A18

Parmentier, G. 2004, MNRAS, 351, 585

Parmentier, G., Goodwin, S. P., Kroupa, P., \& Baumgardt, H. 2008, ApJ, 678, 347

Pasquini, L., Bonifacio, P., Molaro, P., et al. 2005, A\&A, 441, 549

Pasquini, L., Mauas, P., Käufl, H. U., \& Cacciari, C. 2011, A\&A, 531, A35

Pastorello, A., Mattila, S., Zampieri, L., et al. 2008, MNRAS, 389, 113

Peacock, M. B., Maccarone, T. J., Dieball, A., \& Knigge, C. 2011, MNRAS, 411, 487

Pikel'Ner, S. B. 1976, Soviet Ast., 20, 255

Pilachowski, C., Leep, E. M., Wallerstein, G., \& Peterson, R. C. 1982, ApJ, 263, 187

Piotto, G. 2009, in IAU Symp. 258, ed. E. E. Mamajek, D. R. Soderblom, \& R. F. G. Wyse, 233

Piotto, G., Villanova, S., Bedin, L. R., et al. 2005, ApJ, 621, 777

Piotto, G., Bedin, L. R., Anderson, J., et al. 2007, ApJ, 661, L53

Prantzos, N., Charbonnel, C., \& Iliadis, C. 2007, A\&A, 470, 179

Price, N. M., \& Podsiadlowski, P. 1993, in The Globular Cluster-Galaxy Connection, ed. G. H. Smith, \& J. P. Brodie, ASP Conf. Ser., 48, 721

Priestley, W., Ruffert, M., \& Salaris, M. 2011, MNRAS, 411, 1935

Pulone, L., de Marchi, G., Paresce, F., \& Allard, F. 1998, ApJ, 492, L41

Renzini, A. 2008, MNRAS, 391, 354

Rey, S.-C., Lee, Y.-W., Ree, C. H., Joo, J.-M., Sohn, Y.-J., \& Walker, A. R. 2004, AJ, 127, 958

Rey, S.-C., Rich, R. M., Sohn, S. T., et al. 2007, ApJS, 173, 643

Richtler, T., \& Fichtner, H. 1993, in The Globular Cluster-Galaxy Connection, ed. G. H. Smith, \& J. P. Brodie, ASP Conf. Ser., 48, 725

Salaris, M., \& Weiss, A. 1997, A\&A, 327, 107

Salaris, M., Serenelli, A., Weiss, A., \& Miller Bertolami, M. 2009, ApJ, 692, 1013

Shen, Z.-X., Bonifacio, P., Pasquini, L., \& Zaggia, S. 2010, A\&A, 524, L2

Siegel, M. H., Dotter, A., Majewski, S. R., et al. 2007, ApJ, 667, L57

Sills, A., \& Glebbeek, E. 2010, MNRAS, 407, 277

Skillman, E. D. 2008, in IAU Symp. 255, ed. L. K. Hunt, S. Madden, \& R. Schneider, 285

Smith, G. H. 2006, PASP, 118, 1225

Smith, G. H. 2010, PASP, 122, 1171

Smith, V. V., Cunha, K., Ivans, I. I., et al. 2005, ApJ, 633, 392

Sollima, A., Ferraro, F. R., Pancino, E., \& Bellazzini, M. 2005a, MNRAS, 357, 265

Sollima, A., Pancino, E., Ferraro, F. R., et al. 2005b, ApJ, 634, 332

Sollima, A., Ferraro, F. R., \& Bellazzini, M. 2007, MNRAS, 381, 1575

Stanford, L. M., Da Costa, G. S., Norris, J. E., \& Cannon, R. D. 2006, ApJ, 647, 1075

Stothers, R. B., \& Chin, C. 1993, ApJ, 408, L85

Suda, T., Katsuta, Y., Yamada, S., et al. 2008, PASJ, 60, 1159

Tenorio-Tagle, G., Bodenheimer, P., Lin, D. N. C., \& Noriega-Crespo, A. 1986, MNRAS, 221, 635

Thoul, A., Jorissen, A., Goriely, S., et al. 2002, A\&A, 383, 491

Trenti, M., Vesperini, E., \& Pasquato, M. 2010, ApJ, 708, 1598

Tsujimoto, T., Shigeyama, T., \& Suda, T. 2007, ApJ, 654, L139

Ventura, P., D'Antona, F., Mazzitelli, I., \& Gratton, R. 2001, ApJ, 550, L65

Ventura, P., D'Antona, F., \& Mazzitelli, I. 2002, A\&A, 393, 215

Vesperini, E. 1998, MNRAS, 299, 1019

Villanova, S., Piotto, G., King, I. R., et al. 2007, ApJ, 663, 296

Villanova, S., Geisler, D., \& Piotto, G. 2010, ApJ, 722, L18

Whitney, J. H., Rood, R. T., O'Connell, R. W., et al. 1998, ApJ, 495, 284

Wylie-de Boer, E. C., \& Cottrell, P. L. 2009, ApJ, 692, 522

Wylie-de Boer, E., Freeman, K., \& Williams, M. 2010, AJ, 139, 636

Yong, D., \& Grundahl, F. 2008, ApJ, 672, L29 